\title{
Causes systémiques de la chimiose conjonctivale : série de cas
}

\author{
David P. Roncone, \\ OD, FAAO \\ Centre médical \\ de Cleveland, VA \\ Scott A. Anthony, \\ OD, FAAO \\ Centre médical \\ de Cleveland, VA
}

\begin{abstract}
RÉSUMÉ
La chimiose conjonctivale est une affection ophtalmique couramment observée qui présente divers degrés de gravité et un large éventail de symptômes, de signes et d'étiologies sous-jacentes. Bien que la plupart des cas soient de nature oculaire (allergie, infection, irritation), les manifestations atypiques comme la teinte empourprée de la conjonctive, les veines conjonctivales en tire-bouchon et l'œdème périorbital devraient inciter à chercher une cause systémique. Lorsque la chimiose est atypique, l'examen des antécédents médicaux et des médicaments du patient, l'examen physique du cœur et l'examen thoracique ainsi que la prise des signes vitaux (tension artérielle, pouls, poids) sont essentiels à l'identification de la source systémique potentielle. Cet article passe en revue les causes systémiques de la chimiose conjonctivale et présente des exemples de cas qui démontrent les techniques d'évaluation et de prise en charge permettant aux optométristes d'établir une distinction entre la chimiose conjonctivale oculaire et la chimiose conjonctivale systémique.
\end{abstract}

MOTS CLÉS :

chimiose conjonctivale, œedème périorbital, cutané, syndrome de la veine cave supérieure, hypervolémie

\section{INTRODUCTION}

La chimiose conjonctivale, caractérisée par l'œdème de la conjonctive et de la caroncule, est une complication ophtalmique courante qui présente divers degrés de gravité et un large éventail de symptômes, de signes et d'étiologies sous-jacentes. Les caractéristiques cliniques courantes comprennent l'œdème diffus et translucide de la conjonctive bulbaire et de la caroncule, l'apparition de replis ou de crêtes dans le cul-de-sac conjonctival et les papilles de la conjonctive tarsale associée., ${ }^{1,2}$ Les caractéristiques atypiques de la chimiose peuvent comprendre la congestion ou la teinte empourprée de la conjonctive. Pour en établir l'étiologie, il est essentiel d'utiliser les antécédents oculaires, les symptômes et les signes de la biomicroscopie de la lampe à fente (gravité et coloration de la chimiose et signes oculaires connexes). Bien que la plupart des cas de chimiose conjonctivale soient de nature oculaire (allergie, infection, irritation), les manifestations atypiques devraient inciter à chercher une cause systémique. Lorsque la chimiose est atypique, l'examen des antécédents médicaux et des médicaments du patient, l'examen physique du cour et l'examen thoracique ainsi que la prise des signes vitaux (tension artérielle, pouls, poids) sont essentiels à l'identification de la source systémique potentielle. Les maladies systémiques qui accompagnent la chimiose conjonctivale peuvent être graves et même mettre la vie en danger, ce qui souligne l'importance d'identifier cette association. Cet article passe en revue les causes systémiques de la chimiose conjonctivale et présente des exemples de cas qui démontrent les techniques d'évaluation et de prise en charge permettant aux optométristes de faire la distinction entre la chimiose conjonctivale dérivée de l’oil et la chimiose conjonctivale systémique.

\section{RAPPORTS DE CAS}

\section{Cas 1 :}

Un Afro-Américain de 59 ans s'est présenté pour un examen de la vue de routine avec une vision est stable et confortable dans les deux yeux (OU). Les antécédents oculaires du patient sont pertinents pour la dégénérescence 
vitreuse OU, la blépharite OU et la rétinopathie hypertensive légère OU. Il n’y a pas d'antécédents de traumatisme oculaire ou d'intervention chirurgicale, ni d'exposition récente à des allergènes ou d'allergies saisonnières. Les antécédents médicaux sont pertinents pour l'hypertension essentielle bénigne, la fibrillation auriculaire sans récidive après le traitement par une cardioversion en courant continu, l'anévrisme de l'aorte ascendante, les troubles bipolaires, le trait drépanocytaire, le déplacement d'un disque intervertébral, l'adénome du cortex surrénal, les douleurs dorsales chroniques et la polytoxicomanie (alcool, marijuana, cocaïne).

Les médicaments comprennent l'albutérol en inhalateur oral, l'aspirine à $81 \mathrm{mg}$, le carvédilol, le divalproex, l'ibuprofène, l'oméprazole, le citrate de sildénafil et la tamsulosine. Les allergies comprennent les allergies à l'halopéridol, à la lurasidone et aux arachides.

L’examen oculaire a révélé une acuité visuelle corrigée de 20/20 OD et OS. Les pupilles, la motilité extraoculaire, le test de l'écran et la périmétrie par confrontation étaient normaux dans les deux yeux. L'examen physique complet a révélé un œdème de 1+ du bord orbital supérieur et inférieur, l'engorgement veineux de la veine jugulaire droite, la dyspnée, la toux sèche sans expectoration et l'œdème retenant le godet de la cheville droite et de la cheville gauche. On a noté une tension artérielle élevée (160/105 mm Hg) et un pouls normal ( 84 battements par minute). La biomicroscopie de la lampe à fente a révélé des collerettes sur les paupières. La conjonctive a révélé une chimiose avec teinte empourprée de $3+$ de 6 à 9 heures OD et une chimiose rose foncé de 1+ de 3 à 4 heures OS (figure 1). Un arc cornéen OU a été observé avec un temps de bris du film lacrymal de 10 secondes. Le cristallin présentait une opacité corticale de 1+ OU. Les pressions d'aplanation intraoculaires étaient de 16 mm Hg OD et de 14 mm Hg OS à 9 h 55. L'examen du fond d'œil dilaté a révélé un décollement postérieur de la rétine $\mathrm{OU}$ et des vaisseaux sanguins rétiniens tortueux correspondant à une rétinopathie hypertensive OU.

Figure 1 : Cas 1 : Chimiose conjonctivale diffuse secondaire à l'hypervolémie causée par une insuffisance cardiaque aiguë en décompensation avec prolapsus de conjonctive bulbaire sur la marge de la paupière

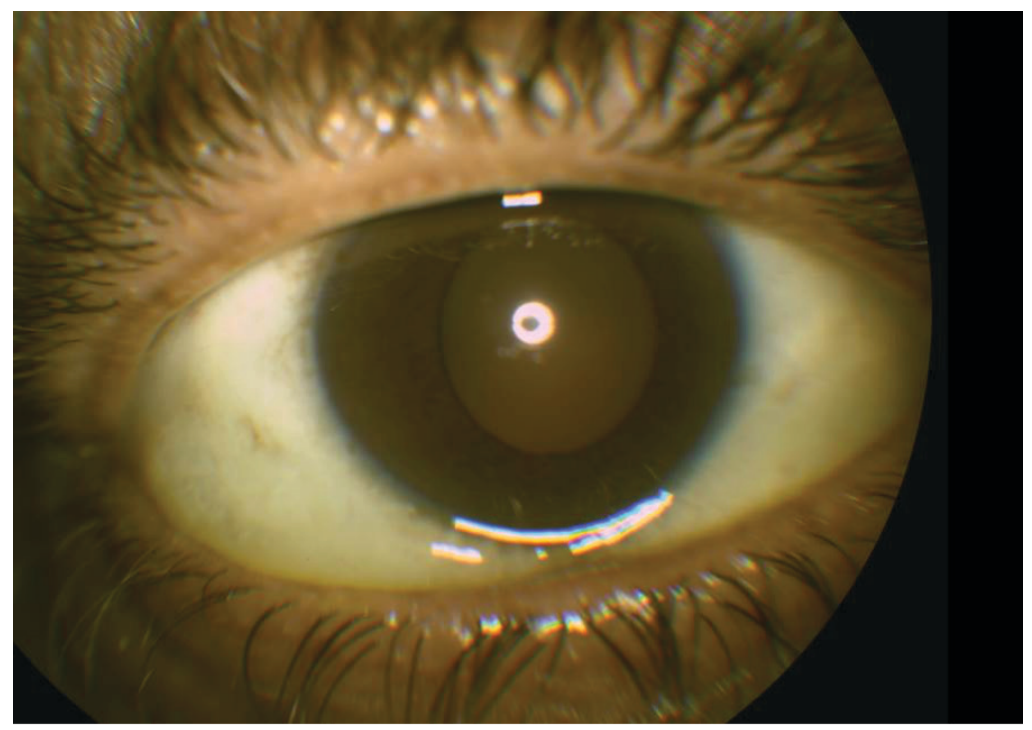

Le patient a reçu un diagnostic de chimiose conjonctivale atypique. En raison des facteurs de risque de cardiopathie chez ce patient (hypertension, fibrillation auriculaire, tabagisme, consommation antérieure d'alcool et de drogues illicites), de signes oculaires (chimiose avec coloration rosée de la conjonctive OU, œdème périorbital OU), des signes d'affection systémique (engorgement veineux de la jugulaire droite et anasarque des chevilles gauche et droite) et des symptômes (dyspnée, toux sèche sans expectoration), une chimiose secondaire à une hypervolémie causée par une insuffisance cardiaque en décompensation a été soupçonnée. Le patient a été envoyé à l'urgence pour qu'il subisse un examen médical plus poussé et des analyses sanguines, y compris une série de tests de la fonction rénale et hépatique et des biomarqueurs cardiaques (peptide natriurétique de type $\mathrm{B}$, troponine-I et créatinine kinase). Les résultats de la série de tests de la fonction rénale et hépatique étaient normaux. Toutefois, des niveaux élevés de peptide natriurétique de type B (2 $277 \mathrm{pg} / \mathrm{ml})$ et de troponine-I $(0,14 \mathrm{pg} / \mathrm{ml})$ ont confirmé un diagnostic d'insuffisance cardiaque décompensée aiguë par un cardiologue. Le patient a été admis à l'hôpital pour une évaluation et un traitement plus poussés. Après que l'on ait stabilisé la tension artérielle du patient (135/90 mm Hg) à 
l'aide d'hydralazine intraveineuse, de furosémide et d'acétaminophène, celui-ci a reçu son congé de l'hôpital et une nouvelle prescription d'atorvastatine calcique, de lisinopril et de spironolactone. Un bilan cardiovasculaire effectué une semaine plus tard, lequel comprenait un angiogramme coronarien et un échocardiogramme transthoracique, a confirmé une insuffisance cardiaque droite et gauche que le patient a choisi de gérer médicalement avec un anticoagulant oral et un antiarythmique. Un mois plus tard, des tests de cardiologie et d'optométrie ont confirmé la stabilité cardiovasculaire et la disparition de la chimiose conjonctivale.

\section{Cas 2 :}

Un Afro-Américain diabétique de 67 ans s'est présenté à son examen annuel de la vue avec une vision stable OU. Il a dit éprouver des démangeaisons de la peau et une enflure indolore de l'œil gauche depuis dix mois, soit depuis qu'il prend $5 \mathrm{mg}$ d'amlodipine tous les jours pour traiter l'hypertension. Ses antécédents oculaires comprennent un dysfonctionnement meibomien, le syndrome de l'œil sec, une légère rétinopathie hypertensive OU, le diabète sucré de type 2 sans complications ophtalmiques OU et de légères cataractes OU. Il n'y a pas d'antécédents de traumatisme oculaire ou d'intervention chirurgicale ni d'exposition récente à des allergènes. Les antécédents médicaux sont pertinents pour l'hypertension, l'hyperlipidémie, le diabète sucré de type 2 avec neuropathie, l'obésité, l'apnée du sommeil, le trouble érectile masculin, l'hypertrophie bénigne de la prostate, la sténose rachidienne de la région lombaire, l'arthrose et le reflux gastro-œsophagien.

Les médicaments du patient comprennent des larmes artificielles, le fosinopril, la térazosine, ls simvastatine, le bésylate d'amlodipine, l'oméprazole, le vardénafil, l'aspirine, le tramadol, la métformine, l'hydrochlorothiazide et cholécalciférol (vitamine D3).

L'examen oculaire a révélé une acuité visuelle corrigée de 20/20 OD et OS. Les pupilles, la motilité extraoculaire, le test de l'écran et la périmétrie par confrontation étaient normaux dans les deux yeux. L'examen physique général a permis de relever un œdème périphérique retenant le godet dans les deux chevilles. Une tension artérielle légèrement élevée $(162 / 91 \mathrm{~mm} \mathrm{Hg})$ et un pouls normal (75 battements par minute) ont été notés. La biomicroscopie de la lampe à fente des paupières a révélé des sécrétions troubles des glandes de Meibomius OU, une mélanose conjonctivale d'origine raciale $\mathrm{OU}$, un ptérygion nasal OU et une légère chalasise conjonctivale OD. L'œil gauche a révélé une chimiose conjonctivale bulbaire translucide de $3+$ allant du limbe au fornix avec conservation du quadrant supérieur OS (figure 2). Le patient a confirmé qu'il dormait habituellement en décubitus sur son côté gauche. La cornée, la chambre antérieure, l'iris et le cristallin OU n'avaient rien d'anormal. Le temps de bris du film lacrymal était de 7 secondes. Les pressions d'aplanation intraoculaires étaient de $15 \mathrm{~mm} \mathrm{Hg} \mathrm{OD} \mathrm{et}$ de $16 \mathrm{~mm} \mathrm{Hg}$ OS. L'examen du fond de l'œil dilaté a révélé la présence de vaisseaux sanguins rétiniens tortueux correspondant à une rétinopathie hypertensive OU.

Figure 2 : Cas 2 : Chimiose conjonctivale diffuse secondaire à une allergie à l'amlodipine avec oedème conjonctival translucide et enflure de la caroncule

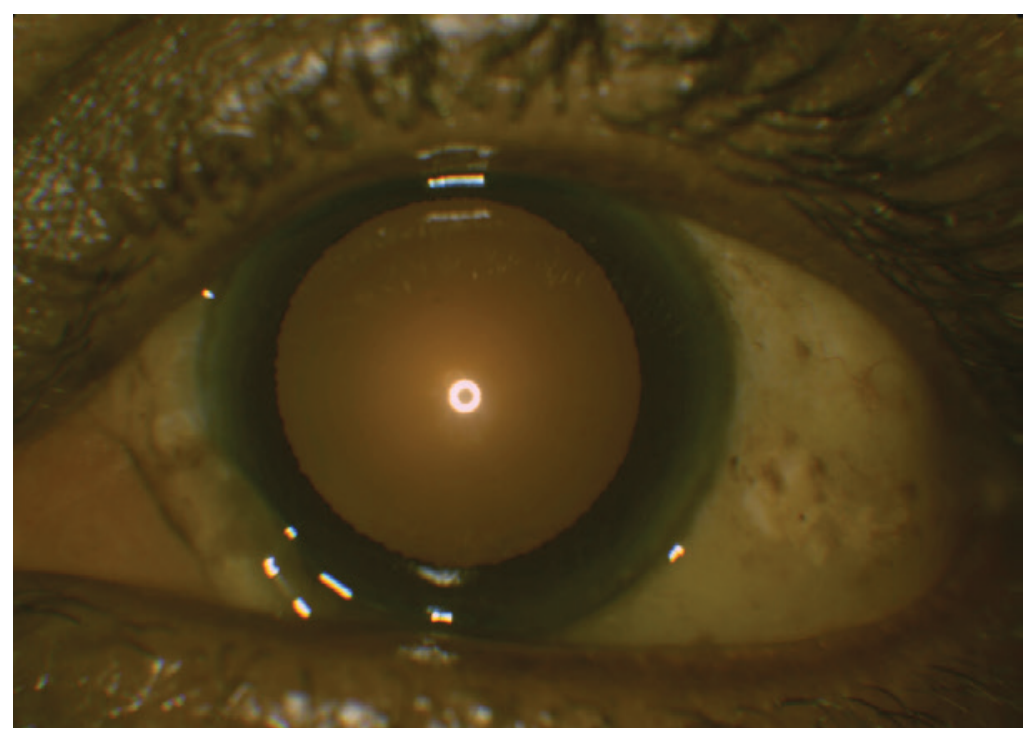


On a conseillé au patient de continuer à appliquer des compresses chaudes 2 f.p.j. OU et des larmes artificielles 3 f.p.j. OU. On lui a dit que sa chimiose conjonctivale nécessitait une consultation non urgente chez un médecin de premier recours en raison d'une allergie présumée à l'amlodipine, surtout après que le patient ait confirmé ressentir des démangeaisons cutanées. On a conseillé au patient de continuer à prendre de l'amlodipine car une réaction d'hypersensibilité à ce médicament aurait constitué un diagnostic d'exclusion. Le patient a également été aiguillé vers un cardiologue pour subir une évaluation en raison de ses facteurs de risque cardiaques (diabète, hypertension, hyperlipidémie et apnée du sommeil). L'évaluation du cardiologue, qui comprenait un angiogramme coronarien, un échocardiogramme transthoracique, une série de tests de la fonction rénale et hépatique et des biomarqueurs cardiaques (peptide natriurétique de type $\mathrm{B}$, troponine-I et créatinine kinase) a révélé une cardiopathie ischémique sans insuffisance cardiaque décompensée aiguë. L'allergie à l'amlodipine a été confirmée en raison de la stabilité cardiopulmonaire et de l'anasarque des chevilles ne retenant pas le godet. L'amlodipine a été abandonnée et remplacée par le carvédilol. Lors du suivi à un mois en cardiologie et de l'examen de la vue, la tension artérielle du patient était bien contrôlée et la chimiose conjonctivale avait disparu.

\section{DISCUSSION}

\section{Anatomie}

Pendant les épisodes de chimiose conjonctivale, la conjonctive bulbaire est principalement touchée. La conjonctive bulbaire est une muqueuse semitransparente et lâchement rattachée qui recouvre le globe antérieur, entre le fornix supérieur et inférieur, et qui rejoint le limbe cornéen ${ }^{3}$. Dans les couches du stroma de la conjonctive bulbaire se trouve une matrice de vaisseaux lymphatiques (sous-muqueuse) et de vaisseaux sanguins (sous-muqueuse profonde) où peuvent s'accumuler les liquides extracellulaires. L'espace entre la conjonctive et le fascia de Ténon, où l'attachementt est lâche, est le lieu où les fluides s'accumulent durant l'inflammation, l'infection et les épisodes vasculaires ${ }^{4}$. Comme la conjonctive palpébrale adhère fermement au tarse, l'œdème est moins prononcé à ce niveau et peut simplement affecter les papilles.

\section{Antécédents oculaires}

Un examen approfondi des antécédents oculaires peut être révélateur chez les patients qui présentent une chimiose conjonctivale du fait que la plupart des cas ont une étiologie oculaire sous-jacente ayant un mécanisme

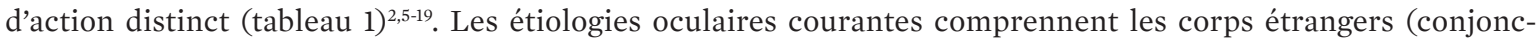
tive, cornée, paupières), les traumatismes mécaniques (frottement des yeux, choc violent, trichiasis, kyste dans la concrétion du tarse, papilles ou follicules), glaucome aigu, infection oculaire (conjonctivite, kératite, sclérite, endophthalmite) ou infection orbitale (cellulite orbitaire), allergie (conjonctivite) et autres troubles oculaires inflammatoires (épisclérite, sclérite, uvéite) ${ }^{12-15,18}$.

Les effets toxiques indésirables des médicaments ophtalmiques, appelés «medicamentosa » ou " kérato-conjonctivite $»^{20}$, peuvent aussi produire une chimiose conjonctivale. De nombreux médicaments ophtalmique en-

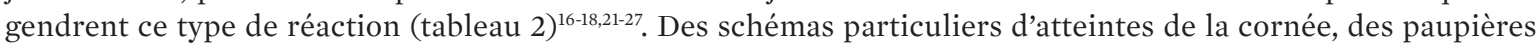
ou de la conjonctive (réactions folliculaires papillaires, amenuisement du symblépharon et/ou du fornix, scarification, hyperémie/induration/écaillage de la peau périoculaire) peuvent contribuer à confirmer cette étiologie (tableau 3) ${ }^{16,172,21,28}$. Une injection dans la conjonctive bulbaire peut s'avérer plus efficace dans la région inférieure que supérieure et une épithéliopathie de la cornée peut mieux réagir au niveau inféronasal en raison de la durée du contact avec les substances pharmaceutiques ${ }^{28,29}$. Les signes cliniques prennent habituellement plusieurs semaines à se développer ${ }^{18}$ et la chimiose conjonctivale unilatérale ou asymétrique n'est pas rare en présence d'une medicamentosa ou autre exposition toxique ${ }^{17}$.

\section{Biomicroscopie de la lampe à fente - signes cliniques}

La biomicroscopie détaillée des lampes à fente est essentielle au diagnostic et à la prise en charge la chimiose conjonctivale. ${ }^{1,2}$ Les principales caractéristiques de l'œdème conjonctival comprennent l'œedème translucide de la conjonctive bulbaire, l'apparition de replis ou de crêtes dans le cul-de-sac conjonctival et les papilles de la conjonctive tarsale associé. L'œè̀me de la caroncule se manifeste par une hyperémie de la caroncule, laquelle paraît parfois sèche. La chimiose conjonctivale bulbaire légère est plus susceptible de présenter des replis et des crêtes répétitifs qui s'étendent au-delà de la jonction mucocutanée de la paupière inférieure, tandis que les cas graves révèlent une conjonctive prolabée sur la paupière inférieure ${ }^{2}$. Bien que la chimiose conjonctivale puisse se manifester unilatéralement, une atteinte bilatérale et symétrique pourrait indiquer une étiologie systémique. La conjonctive a un aspect gélatineux, elle est soit pâle soit incolore, soit rougeâtre ${ }^{1}$. Les chimioses conjonctivales de couleur pâle ou incolores sont habituellement attribuables à des étiologies liées à une congestion non veineuse ${ }^{1}$, comme on le voit dans les traumatismes oculaires, 
les infections oculaires et les conditions inflammatoires, les brûlures chimiques et les réactions d'hypersensibilité de contact. Les chocs violents et les traumatismes chimiques sont des urgences oculaires qui nécessitent une consultation en ophtalmologie lorsqu'une chimiose conjonctivale est soupçonnée d'être accompagnée d'une rupture de la conjonctive scléroticale ou d'une brûlure chimique alcaline ${ }^{5}$. La chimiose conjonctivale qui prend une teinte rougeâtre et qui s'accompagne d'une dilatation veineuse importante est plus susceptible d'être causée par un blocage veineux systémique ou une congestion ${ }^{1}$. La chimiose conjonctivale montrant des veines en tire-bouchon laisse supposer un écoulement veineux rétrograde appelé « artérialisation». Quatre-vingt-dix pour cent des patients qui présentent une artérialisation des veines conjonctivales ont une fistule des sinus caverneux carotidiens ${ }^{30}$. Les paupières doivent être retournées pendant la biomicroscopie avec lampe à fente pour exclure une étiologie mécanique ou traumatique (corps étrangers, trichiasis, kyste dans la concrétion du tarse et de la conjonctive palpébrale, papilles ou follicules). Il peut être utile de vérifier les sensibilités cornéennes si l'on soupçonne un herpès oculaire et une kératopathie neurotrophique.

\section{Symptomatologie}

En l'absence d'une étiologie oculaire sous-jacente à la chimiose conjonctivale, les antécédents complets du patient, l'examen de la symptomatologie et l'identification de toute réaction d'hypersensibilité aux médicaments, aux aliments, aux produits chimiques, aux solutions pour lentilles cornéennes, aux produits cosmétiques (pour la peau et les cheveux) ainsi que de la consommation de drogues illicites peuvent aider à établir si l'étiologie est de nature systémique (tableau $4 \mathrm{a}-\mathrm{e}^{6,18,30-52}$ et tableau $5^{2,6,18,3,33,40,42,49,53-58}$.)

L'acuité ou la chronicité des symptômes peut aider à différencier la cause localisée de la chimiose conjonctivale oculaire de la cause systémique. L'apparition soudaine d'une chimiose conjonctivale est habituellement révélatrice d'une réaction d'hypersensibilité (tableau 2) 216-18,21-27. L'apparition d'une chimiose conjonctivale sous-aiguë ou chronique peut avoir de nombreuses étiologies, incluant une réaction localisée des tissus oculaires (thyroïdopathie affectant les yeux, conjonctivite consécutive à une allergie chronique, intervention chirurgicale visant les paupières ou les yeux, traumatisme), hausse de la perméabilité vasculaire systémique (états allergiques, infections comprenant la méningite, vascularite), hausse de la pression veineuse (syndrome de la veine cave supérieure, insuffisance cardiaque) et baisse de la pression oncotique plasmatique (syndrome néphrotique, néphropathie) ${ }^{2,18,59}$.

\section{ÉTIOLOGIES SYSTÉMIQUES DE LA CHIMIOSE CONJONCTIVALE}

\section{Réactions d'hypersensibilité}

De nombreux médicaments systémiques ont été impliqués dans la chimiose conjonctivale liée à l'hypersensibilité (tableau 2$)^{16-18,21-27}$. Bien que les réactions d'hypersensibilité d'origine médicamenteuse représentent $36,2 \%$ de l'anaphylaxie, les allergies alimentaires demeurent la cause la plus courante (49,7\%) et la consommation récente d'aliments doit être examinée ${ }^{33}$. Les signes oculaires peuvent présenter des signes et des symptômes dermatologiques concomitants. Les réactions cutanées (démangeaisons intenses, bouffées vasomotrices, œdème des yeux, des lèvres, de la langue et de la luette) et l'urticaire (éruptions cutanées parfois accompagnées d'œedème dermique superficiel) montrent les symptômes d'hypersensibilité les plus courants. Leur prévalence est plus élevée quand elles sont associées aux aliments (90,9\% et $86,9 \%$, respectivement) par rapport aux médicaments $(69,4 \% \text { et } 66,7 \% \text {, respectivement })^{33}$. Bien que les manifestations de dyspnée soient égales dans les deux groupes, les symptômes respiratoires (respiration sifflante, stridor, hypoxémie) sont plus courants en présence d'une allergie alimentaire tandis que les symptômes cardiovasculaires (syncope, hypotension, incontinence urinaire, malaise pulmonaire) sont plus courants en présence d’une allergie aux médicaments ${ }^{33}$.

\section{Réactions non attribuables à une hypersensibilité}

Les étiologies systémiques de la chimiose conjonctivale qui ne sont pas attribuables aux réactions d'hypersensibilité comprennent les atteintes du sinus caverneux (fistule ou thrombose carotido-caverneuse), la thyroïdopathie (hyperthyrö̈die, maladie de Graves), le syndrome de la veine cave supérieure et l'hypervolémie [avec ses diverses conditions systémiques sous-jacentes] (tableau $4 \mathrm{~b}-\mathrm{e})^{6,18,30-52}$.

\section{Atteintes du sinus caverneux}

Une atteinte du sinus caverneux (fistule ou thrombose carotido-caverneuse) peut se présenter avec une chimiose conjonctivale, en plus de nombreux déficits des nerfs crâniens.

La fistule carotido-caverneuse est une communication anormale entre le système veineux du sinus caverneux et l'artère carotide interne ou ses ramifications vers les méninges ou, encore, dans les ramifications de l'artère carotide externe vers les méninges ${ }^{60}$. La chimiose conjonctivale est manifeste chez $42 \%$ des patients ayant une fistule carotido-caverneuse et se caractérise par l'observation pathognomonique d'une artérialisation en tire-bouchon des 
veines conjonctivales $(93 \%)^{30}$. D'autres signes cliniques attribuables à l'effet de congestion de la circulation sanguine anormale dans le sinus caverneux comprennent l'exophtalmie ( $84 \%)$, la baisse de l'acuité visuelle (43\%), la paralysie des nerfs crâniaux (52 \%) et le souffle $(28 \%)^{30}$.

La thrombose carotido-caverneuse est un caillot de sang (thrombus ou embolie) d'origine aseptique (intervention chirurgicale, traumatisme) ou septique (sinusite, otite, infection œedontogène, furoncle facial, érysipèle) qui se déplace vers les sinus caverneux $\mathrm{x}^{61}$. Les signes d'atteinte aiguë émanant d'une congestion ou du blocage du système veineux comprennent l'exophtalmie, la ptosis et la chimiose ${ }^{32}$. Ces patients sont plus susceptibles d'avoir une paralysie des nerfs crâniens que les patients ayant une fistule carotido-caverneuse patients (80-100 \% contre $52 \%$ ) et le nerf abducens est affecté la plupart du temps $(73 \%)^{32}$. Cependant, les principaux symptômes de différenciations que sont l'œedème périorbital, les maux de tête, la léthargie et les troubles de la conscience (50-80 \%) sont des effets types de la thrombose carotido-caverneuse ${ }^{32}$.

\section{Hyperthyroïdie}

L'hyperthyroïdie peut entraîner une chimiose conjonctivale secondaire à l'accumulation de glycosaminoglycanes dans les tissus conjonctifs, ce qui entraîne un œedème et une inflammation des muscles extraoculaires, des tissus conjonctifs orbitaux et des tissus adipeux, une augmentation du volume orbital ainsi qu'une diminution du drainage veineux et lymphatique ${ }^{49,53}$. La recherche d'une hyperthyroïdie associée devrait tenir compte de l'âge du patient. Les patients plus jeunes $(<50$ ans) ressentent habituellement davantage de symptômes que les patients plus âgés ( $>70$ ans), bien que les symptômes types soient les mêmes dans les deux groupes : tachycardie ( $96 \%$ contre $71 \%$ ), fatigue $(84 \% \text { contre } 56 \% \text { ) et perte pondérale (51\% contre } 50 \%)^{33,34}$. En outre, les patients plus jeunes sont plus susceptibles d'éprouver d'autres symptômes dont des symptômes neurologiques, la dyspnée et la polydipsie (tableau 4) $)^{6,18,30-52}$. Vingt-cinq pour cent des patients atteints de thyroïdopathie ont une orbitopathie d'origine thyroïdienne ${ }^{35,36}$ et éprouvent les symptômes courants suivants : douleur (30\%), larmoiement (20,8\%), diplopie lors de la manifestation initiale (16,6 \%), photophobie $(15,8 \%)$ et vision brouillée $(7,5 \%)^{37}$.

\section{Syndrome de la veine cave supérieure}

L’obstruction ou la compression de la veine cave supérieure, grande veine dans laquelle le sang désoxygéné transite des extrémités des membres supérieurs, de la tête, de la nuque et du thorax jusqu'au ventricule droit, peut affaiblir le drainage veineux, état appelé «syndrome de la veine cave supérieure » ${ }^{40}$. L’un de ses signes les plus courants est l'œedème du visage ou de la nuque $(82 \%)^{40}$, lequel s'aggrave en décubitus. Les patients mentionnent parfois aussi une enflure des extrémités des membres supérieurs $(68 \%)$, de la dyspnée $(66 \%)$ et de la toux $(50 \%)^{40}$. Soixante pour cent des cas de syndrome de la veine cave supérieure ont une étiologie de malignité, plus précisément un cancer broncho-pulmonaire (à petites cellules ou non à petites cellules) (46\%), un lymphome (8\%) ou des tumeurs de cellules germinales $(8 \%)^{40}$. Le syndrome de la veine cave supérieure a d'autres étiologies dans $40 \%$ des cas, par exemple le matériel médical (dispositifs intravasculaires, cathéters de dialyse, fils de stimulateurs cardiaques, cathéter Hickman) et la médiastinite fibreuse (secondaire à un lymphome, à l'histoplasmose, à la tuberculose, à un anévrisme aortique d'origine syphilitique $)^{40}$. Soixante et onze pour cent de ces étiologies bénignes sont secondaires à l'utilisation d'un dispositif médical intravasculaire, laquelle constitue la cause la plus courante ${ }^{40}$.

\section{Hypervolémie}

L'hypervolémie, qui se traduit par une surcharge liquidienne, est une affection où le sang contient un excès de liquides, principalement du plasma sanguin, du sel et de l'eau. Les affections associées à l'hypervolémie comprennent l'insuffisance cardiaque, le syndrome néphrotique et les maladies hépatiques, chacune ayant des signes et des symptômes distinctifs.

Lors de l'évaluation des patients atteints d'insuffisance cardiaque hypervolémique, l'examen physique a une spécificité supérieure et sensibilité moindre que les antécédents du patient ${ }^{41}$. Le symptôme général le plus courant est la (87-93\%) dont la dyspnée d'effort est le type le plus courant $(86-97 \%)^{41}$. D'autres symptômes plus prévalents comprennent l'œè̀me (35-70 \%), la distension de la veine jugulaire (5-54 \%), les râles (25-45\%) et le bruit de galop du cœur (1-26\% \% .

Le syndrome néphrotique est un trouble rénal souvent idiopathique et caractérisé par de l'œdème périphérique, une forte protéinurie, de l'hypoalbuminémie et de l'hyperlipidémie ${ }^{42-44}$. Le diabète sucré de type 2 et le lupus érythémateux systémique sont des affections les plus souvent associées aux causes systémiques du syndrome néphrotique $^{43,44}$. Les signes cliniques comprennent l'œdème périorbital, plus accentué le matin, l'œdème des jambes retenant le godet en fin de journée ${ }^{42}$, l'œdème de l'abdomen et des parties génitales ${ }^{42}$, l'urine spumeuse attribuable à une protéinurie ${ }^{42}$, la coloration blanche des ongles avec ou sans bandes blanches (lignes de Muehrcke) attribuable à une hypoalbuminémie ${ }^{42}$ et les xanthomes cutanés attribuables à un taux élevé de cholestérol sérique ${ }^{58}$. Certains patients éprouvent un malaise ${ }^{42}$. Les patients diabétiques atteints de syndrome néphrotique présentent les symptômes clas- 
siques que sont la perte pondérale, la polyurie, la polydipsie et la polyphagie. Les patients atteints de lupus érythémateux systémique sont le plus souvent atteints d'arthrite et/ou d'arthralgie (86\%), d'érythème en papillon (61 \%) et d'anémie (55\%), suivis de la photosensibilité (48\%), de la fièvre (43\%), d'ulcères de la bouche (43\%), de maux de tête $(36 \%)$, de la triade de la fatigue, des malaises et de la faiblesse (35\%) et d'alopécie (35\% ${ }^{45}$.

Le syndrome néphrotique associé à une néphropathie peut causer des symptômes non spécifiques de malaise ou d'inconfort abdominal ${ }^{46}$. Bien que l'examen pertinent des antécédents d'alcoolisme puisse aider l'optométriste à établir un diagnostic de stéatose hépatique, les patients non alcooliques sont plus difficiles à diagnostiquer du fait que la plupart de ces cas sont détectés de manière fortuite lorsque le taux d'enzymes hépatiques est élevé ou au moyen des tests sanguins habituels ${ }^{46}$. Certains patients peuvent avoir noté la présence d'une masse abdominale ou d'un renflement dorso-cervical, lesquels sont associés à une stéatose hépatique non alcoolique ${ }^{47}$.

DIAGNOSTIC DIFFÉRENTIEL

La chalasise conjonctivale et la lymphangiectasie conjonctivale peuvent avoir des caractéristiques qui ressemblent à celles d'une chimiose conjonctivale.

La chalasise conjonctivale est une atteinte bilatérale associée au vieillissement dans laquelle des prolapsus de tissu conjonctival redondant se forment vers l'avant, au-delà de la marge de la paupière, possiblement en obstruant le méat lacrymal inférieur ${ }^{63}$. La conjonctive redondante est habituellement localisée vers les tempes et le centre, quelques millimètres sous le limbe inférieur ${ }^{64}$, tandis que la chimiose conjonctivale s'étend à n'importe quel quadrant. Les cas bénins de chalasise conjonctivale sont généralement asymptomatiques, tandis que les cas graves sont plus susceptibles d'entraîner une obstruction du méat lacrymal et une instabilité du film lacrymal associées à la sensation d'avoir un corps étranger et d'un épiphore. Ces complications résultent souvent d'une perturbation mécanique du ménisque lacrymal et de l'altération du drainage nasolacrymal ${ }^{64}$.

La chalasise conjonctivale est une maladie acquise rare caractérisée par une dilatation unilatérale ou bilatérale des vaisseaux sanguins de la conjonctive habituellement temporaire ${ }^{65}$. Cet état représente un lymphœème secondaire causé par la rupture ou l'obstruction du flux lymphatique dans la conjonctive ${ }^{65}$. Elle se manifeste par l'élargissement diffus des vaisseaux lymphatiques de la conjonctive (qui donnent l'apparence d'une chimiose) ou par la dilatation focalisée des vaisseaux qui ressemblent alors à des kystes. On parle parfois de « chaîne de perles » ou de « saucissons » ${ }^{6}$, selon la présence ou l'absence de sang. Bien que le diagnostic de cet état soit le plus souvent basé sur l'apparence, il convient de pratiquer une biopsie quand il devient récurrent ${ }^{65}$. Une tomographie par cohérence optique du segment antérieur peut aider les cliniciens à éviter la biopsie et confirmer la présence de vaisseaux lymphatiques dilatés ${ }^{66}$. Bien que l'étiologie soit inconnue, la lymphangiectasie peut être associée à un traumatisme antérieur, à d'autres maladies (ptérygion, néoplasme) ou à des causes iatrogènes (intervention chirurgicale oculaire, radiothérapie) ${ }^{65}$. Les patients atteints de lymphangiectasie se plaignent habituellement d'irrégularités épibulbaires comprenant l'injection, l'irritation et l'épiphore ${ }^{65}$.

\section{EXAMEN EXTERNE}

Une chimiose conjonctivale soupçonnée attribuable à un problème systémique nécessite l'examen physique du patient, y compris des yeux et de leurs annexes, de la tête et de la nuque, des bras et des jambes, du système tégumentaire et des cheveux, du système pulmonaire et de l'appareil cardiovasculaire. Si l'on soupçonne une association systémique particulière à la suite d'un examen superficiel, la coordination des soins avec d'autres spécialistes est importante (tableaux 4 et 5) $)^{2,6,18,30-58}$.

\section{Annexes de l'œil}

Les cas de chimiose conjonctivale accompagnée d'une rétraction de la paupière et d'œdème, de tissu scléral apparent et d'une exophtalmie sont habituellement révélateurs d'une hyperthyroïdie. La combinaison d'une exophtalmie et d'une la chimiose conjonctivale devrait mener à la recherche d'une cellulite orbitale et du syndrome des sinus caverneux, en particulier si des déficits sont observés lors des tests du muscle extraoculaire et de la pupille.

\section{Appareil cardiovasculaire et système pulmonaire}

Les signes vitaux (température, poids, pouls, tension artérielle) et l'auscultation du cour et des poumons sont des signes (bruit de galop, râles, respiration sifflante, stridor) qui peuvent aider à établir l'étiologie systémique de la chimiose.

La cellulite orbitale doit être exclue chez les patients fébriles ou dont la température orale est supérieure à $37,5^{\circ} \mathrm{C}$ $\left(99,5^{\circ} \mathrm{F}\right)$. La cellulite orbitale peut progresser et causer des signes de douleur lors du mouvement oculaire, un déficit pupillaire afférent et un œdème périorbital. 
Lae perte de poids involontaire est indicatrice d'une hyperthyroïdie ou d'une malignité tandis que la prise de poids suggère une hypervolémie ${ }^{41}$.

La tachycardie pourrait indiquer une hyperthyroïdie ou une insuffisance cardiaque, selon les signes et symptômes connexes $^{34,41}$. Le bruit de galop (rythme cardiaque à 3 ou 4 temps) est perçu chez jusqu'à $26 \%$ des patients qui ont une insuffisance cardiaque, en plus des râles (crépitement à l'inhalation) chez 25 à $45 \%$ des patients ${ }^{41}$.

L'hypotension (tension artérielle inférieure à $90 / 60 \mathrm{~mm} \mathrm{Hg}$ ) et le stridor (bruit de sifflement aigu lors de l'inhalation) évoquent un diagnostic possible d'hypersensibilité aux médicaments ou aux aliments ${ }^{33}$.

La dyspnée observée lors de l'examen externe peut être secondaire à une réaction d'hypersensibilité, à l'hyperthyroïdie, à l'insuffisance cardiaque, au syndrome de la veine cave supérieure ou à diverses étiologies d'hypervolémie sous-jacentes $^{33,40,41,44}$. L'évaluation du signe de Pemberton chez les patients, c.-à-d. l'apparition d'une congestion faciale et d'une cyanose lorsqu'un patient élève les deux bras, est spécifique d'un syndrome latent du syndrome de la veine cave supérieure ${ }^{67}$.

\section{Tête et nuque}

Il est essentiel d'évaluer la tête et la nuque du patient pour écarter la possibilité d'une lymphadénopathie et d'une distension des veines jugulaires du fait que les lymphomes, les thyroïdopathies, les cancers bronchopulmonaires, la tuberculose, le lupus érythémateux systémique et les anévrismes aortiques d'origine syphilitique peuvent être associés à une lymphadénopathie et une maladie cardiopulmonaire à la distension des veines jugulaires. Tous ces symptômes peuvent se manifester en présence d'une chimiose conjonctivale d'étiologie systémique.

\section{Système tégumentaire et cheveux}

L'examen du système tégumentaire du patient pour déceler des manifestations cutanées comme l'alopécie (perte de cheveux), les éruptions cutanées (changements de l'apparence de la peau, y compris de la texture et/ou de la couleur) et l'œdème peut aider le praticien à déterminer les étiologies systémiques de la chimiose conjonctivale (tableau 6) ${ }^{18,31,33,3,42,45,48,49,51,52,68-70}$.

L’hyperthyroïdie, le lupus érythémateux systémique et la syphilis secondaire et tertiaire peuvent rendre les cheveux épars et cassants et/ou causer une perte de cheveux (alopécie) du cuir chevelu, des sourcils et des cils ${ }^{48,71}$.

La jaunisse peut être présente avec diverses pathologies systémiques, notamment les maladies hépatiques, la tuberculose, l'histoplasmose, la syphilis, les lymphomes hodgkiniens et non hodgkiniens, le syndrome cardio-rénal et le lupus érythémateux systémique ${ }^{51,71}$. Les xanthomes cutanés sont habituellement observés chez les patients ayant un syndrome néphrotique et de l'hypercholestérolémie ${ }^{42}$. Les patients dont les ongles sont de couleur blanchâtre, avec ou sans bandes blanches (lignes de Muehrcke), ont habituellement un syndrome néphrotique sous-jacent ${ }^{42}$. Les éruptions cutanées sont plus susceptibles d'être attribuables à une réaction d'hypersensibilité ou à un lupus érythémateux systémique. Les réactions d'hypersensibilité se manifestent habituellement par de l'urticaire qui forme des bosses rougeâtres ou des plaques pâles accompagnées d'œè̀me. Ces réactions sont plus souvent associées aux aliments qu'aux médicaments $(85,9 \% \text { contre } 66,7 \%)^{33}$. Les éruptions en forme de papillon du lupus érythémateux systémique sont présentes dans les régions malaires du visage $(61 \%)^{45}$.

L'unilatéralité, le type (retenant le godet ou non), l'acuité et l'emplacement de l'œedème déterminés lors de l'examen externe peuvent aider à établir l'étiologie. Les patients qui dorment en position allongée latérale peuvent présenter, sur le côté où ils se couchent, une accumulation ipsilatérale de liquides interstitiels, par exemple dans un bras ou dans une jambe ou, encore, dans la conjonctive ${ }^{52}$. Un œedème retenant le godet, défini par l'indentation des tissus qui se prolonge après l'application et le retrait d'une pression, peut être observé chez les patients souffrant d'insuffisance cardiaque, de syndrome néphrotique et de néphropathie ou qui prennent des inhibiteurs calciques ${ }^{52}$. L'oedème ne retenant pas le godet, défini par l'indentation des tissus qui se résorbe après l'application et le retrait d'une pression, peut être observé chez les patients souffrant d'un lymphome, d'hyperthyroïdie et du syndrome de la veine cave supérieure ${ }^{52}$.

L’apparition soudaine d'un œdème dans les couches dermiques plus profondes, appelé œedème angioneurotique, est habituellement attribuable à une réaction d'hypersensibilité. Il peut apparaître n'importe où (c.-à-d. sur le visage, la langue, le larynx, l'abdomen, les bras et les jambes) et est plus souvent associé à une réaction d'hypersensibilité aux aliments $(23,2 \%)$ qu'à une réaction aux médicaments $(18,1 \%)^{33}$.

L’œdème buccal (de la langue, des lèvres et de la luette) accompagné de démangeaisons et de bouffées vasomotrices est aussi généralement dû à une réaction d'hypersensibilité; il est plus souvent présent dans les réactions d'hypersensibilité associées aux aliments que dans celles associées aux médicaments $(90,9 \% \text { contre } 69,4 \%)^{33}$. 
Un œè̀me périorbital est habituellement observable chez les patients qui ont une thrombose carotido-caverneuse (80 à $100 \%$ ), une hypersensibilité ou une allergie, une hyperthyroïdie ou un syndrome néphrotique ${ }^{32}$. Les patients atteints du syndrome néphrotique présentent habituellement un œdème périorbital plus notable le matin, un œdème des jambes et de l'abdomen retenant le godet en fin de journée un œedème des parties génitales en fin de journée $e^{42}$.

Chez 1 à $5 \%$ des patients atteints de la maladie de Graves, on observe un myxœedème prétibial ne retenant pas le godet $^{49,52}$. Bien que l'on observe une enflure prémalaire et de la joue chez les patients de moins de 50 ans atteints d'hyperthyroïdie, cette manifestation est rare $(1,84 \%)^{39}$.

L'œdème commun observé avec le syndrome de la veine cave supérieure comprend l'œdème du visage et de la nuque (82\%) et des extrémités des membres supérieurs $(68 \%)$, la grande majorité des patients atteints ayant un cancer bronchopulmonaire ou un dispositif médical intravasculaire ${ }^{40}$. L'œdème du visage chez les patients atteints de cancer du poumon est prononcé le matin et se résorbe en soirée.

Un œedème généralisé, l'anasarque, est observé chez 35 à $70 \%$ des patients atteints d'hypervolémie ${ }^{41}$. On l'observe également chez les patients qui ont une réaction d'hypersensibilité, comme c'est le cas du patient chez qui l'amlodipine a provoqué une anasarque.

L’odème abdominal est habituellement secondaire au syndrome néphrotique ou à une néphropathie ${ }^{43}$. Une néphropathie peut se présenter avec un œdème abdominal, facial et périphérique.

L’œdème périphérique des bras et des jambes est habituellement attribuable à une hypervolémie (insuffisance cardiaque, syndrome néphrotique, affection hépatique ${ }^{52}$.

\section{TRAITEMENT ET PRISE EN CHARGE}

Le traitement dépend de l'étiologie (bactérienne, allergique, virale, inflammatoire) et comprend des interventions chirurgicales, le retrait de corps étrangers ou de kystes dans la paupière et/ou l'amorçage d'un traitement par des antibiotiques ophtalmiques et oraux, des antiviraux, des antiallergiques, des anti-inflammatoires et divers médicaments oraux et intraveineux.

La prise en charge de la chimiose conjonctivale est déterminée par l'évolution de la maladie (c.-à-d. aiguë, subaiguë, chronique), l'étiologie et l'urgence de mettre en œuvre le plan de traitement. Les étiologies oculaires nécessitent habituellement une coordination des soins non urgente, à moins que la chimiose soit secondaire aux troubles oculaires menaçant la vue (pénétration de corps étrangers oculaires, glaucome aigu, cellulite orbitale, rupture de la sclérotique ou énophtalmie). Lorsqu'on soupçonne une rupture de la sclérotique ou une brûlure chimique alcaline, il faut consulter sans tarder un ophtalmologiste. Les cas de cellulite orbitale requièrent le renvoi immédiat du patient vers le service des urgences pour évaluer son état et lui faire subir une tomographie orbitale avec ou sans contraste et où on lui administrera des antibiotiques intraveineux. Il pourrait aussi être redirigé vers un infectiologue. Quand toutes les étiologies oculaires sont exclues, il s'avère crucial de corréler les résultats de la biomicroscopie de la lampe à fente avec les antécédents du patient, ses symptômes et les résultats de l'examen externe. Cela évite la réalisation de tests coûteux dont les résultats ne sont pas garantis ni nécessairement révélateurs pour trouver une étiologie systémique (tableau 3) ${ }^{16,17,21,28}$. Une chimiose que l'on pense attribuable à une thrombose ou à une fistule carotido-caverneuse ou, encore, à une insuffisance cardiaque aiguë en décompensation nécessite le renvoi immédiat et le jour même du patient au service des urgences pour lui faire

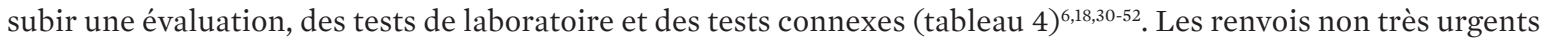
vers d'autres spécialistes nécessitent une coordination des soins pour qu'une évaluation, des tests de laboratoire et des tests connexes appropriés soient effectués afin de confirmer ou d'infirmer les états systémiques liés aux résultats de l'examen de la vue.

\section{CONCLUSION}

La chimiose conjonctivale est fréquente dans un contexte de soins oculovisuels primaires et présente un large éventail d'associations et divers degrés de gravité. Bien que la plupart des cas aient une étiologie oculaire, déterminée par les antécédents et l'examen, ceux dont l'étiologie est systémique exigent l'examen approfondi des antécédents et de la symptomatologie, un examen physique (évaluation de la distension de la veine jugulaire, de l'œdème retenant ou ne retenant pas le godet, de la lymphadénopathie, des éruptions cutanées, de la jaunisse et de l'alopécie) et une évaluation médicale (pouls, TA, poids, auscultation du cœur et du thorax). Dans ce rapport, deux cas de chimiose conjonctivale avec des étiologies différentes ont mené à un aiguillage vers des médecins spécialistes pour gérer et traiter les problèmes systémiques associés. • 
Tableau 1 : Étiologies oculaires de la chimiose conjonctivale et mécanismes d'action correspondants ${ }^{2,5-19}$

\begin{tabular}{|c|c|c|c|}
\hline \multicolumn{2}{|l|}{ Étiologie } & \multicolumn{2}{|l|}{ Mécanisme d'action de la Chimiose } \\
\hline \multicolumn{2}{|c|}{$\begin{array}{l}\text { Traumatisme oculaire }{ }^{5,11} \\
\text { (y compris un traumatisme orbito-facial) }\end{array}$} & $\begin{array}{l}\text { Aiguë : } \\
\text { Réaction inflammatoire provoquée par la } \\
\text { libération de l'histamine, de la sérotonine et de } \\
\text { la bradykinine accompagnée d'une migration des } \\
\text { cellules polymorphonucléaires, laquelle provoque } \\
\text { la dilatation des artérioles, la hausse du gradient } \\
\text { de pression entre les capillaires artériolaires et } \\
\text { les veinules ainsi que l'extravasation du liquide } \\
\text { intravasculaire et œedème de vasodilatation } \\
\text { consécutif }^{5}\end{array}$ & $\begin{array}{l}\text { Chronique: } \\
\text { Blocage du système } \\
\text { lymphatique attribuable } \\
\text { à une scarification ou à } \\
\text { une altération du drainage }_{\text {lymphatique }^{19}}\end{array}$ \\
\hline \multicolumn{2}{|l|}{ Chirurgie oculaire $^{7}$} & \multicolumn{2}{|c|}{$\begin{array}{l}\text { Blocage du système lymphatique attribuable à une scarification ou à une altération } \\
\text { du drainage lymphatique }\end{array}$} \\
\hline $\begin{array}{l}\text { Infection du } \\
\text { segment antérieur }\end{array}$ & $\begin{array}{l}\text { Conjonctivite; } \\
\text { kératite; sclérite; } \\
\text { énophtalmie } \\
\text { (bactérienne ou } \\
\text { virale) } \\
6,14\end{array}$ & \multicolumn{2}{|c|}{$\begin{array}{l}\text { Dilatation des artérioles, hausse du gradient de pression entre les capillaires } \\
\text { artériolaires et les veinules et extravasation du liquide intravasculaire et oedème } \\
\text { vasodilataire consécutif }{ }^{18}\end{array}$} \\
\hline \multirow{3}{*}{$\begin{array}{l}\text { Inflammation du } \\
\text { segment antérieur }\end{array}$} & $\begin{array}{l}\text { Conjonctivite } \\
\text { allergique saisonnière } \\
\text { ou permanente ou } \\
\text { kératite }^{6,12}\end{array}$ & \multicolumn{2}{|c|}{$\begin{array}{l}\text { Réaction d'hypersensibilité de type I (anaphylactoïde) entraînant une libération } \\
\text { d'histamine, de sérotonine et de bradykinine, accompagnée d'une migration de } \\
\text { cellules polymorphonucléaires, laquelle provoque la dilatation des artérioles, la } \\
\text { hausse du gradient de pression entre les capillaires artériolaires et les veinules } \\
\text { ainsi que l'extravasation du liquide intravasculaire et un œedème de vasodilatation } \\
\text { consécutif',18 }\end{array}$} \\
\hline & $\begin{array}{l}\text { Conjonctivite } \\
\text { medicamentosa ou } \\
\text { kératite }^{16,17}\end{array}$ & \multicolumn{2}{|c|}{$\begin{array}{l}\text { Réponse d'hypersensibilité de type IV (à médiation cellulaire) où les lymphocytes T } \\
\text { auxiliaires (CD4) reconnaissent un antigène et déclenchent la libération } \\
\text { d'interleukines, de cytokines et d'enzymes, ce qui entraîne l'extravasation de fluides } \\
\text { intravasculaires et un œdème de vasodilatation consécutifí,18 }\end{array}$} \\
\hline & $\begin{array}{l}\text { Épisclérite; sclérite; } \\
\text { uvéite }^{8}\end{array}$ & \multicolumn{2}{|c|}{$\begin{array}{l}\text { Dilatation des artérioles, hausse du gradient de pression entre les capillaires } \\
\text { artériolaires et les veinules et extravasation du liquide intravasculaire et œème } \\
\text { vasodilataire consécutif }\end{array}$} \\
\hline \multicolumn{2}{|l|}{ Glaucome aigu $^{23}$} & \multicolumn{2}{|l|}{ Nombreux mécanismes selon l'étiologie } \\
\hline \multirow{2}{*}{ Maladie orbitale ${ }^{9}$} & $\begin{array}{l}\text { Infection orbitale } \\
{\text { (cellulite) })^{13}}\end{array}$ & \multicolumn{2}{|c|}{$\begin{array}{l}\text { Infection des sinus ou des voies respiratoires supérieures se propageant vers l'orbite } \\
\text { et causant une infection et une inflammation des tissus oculaires postérieurs à la } \\
\text { cloison orbitale }\end{array}$} \\
\hline & $\begin{array}{l}\text { Pseudotumeur ou } \\
\text { malignité orbitale }\end{array}$ & \multicolumn{2}{|c|}{ Congestion ou blocage du système lymphatique ou veineux ${ }^{11}$} \\
\hline
\end{tabular}


Tableau 2 : Médicaments oculaires et systémiques qui induisent des chimioses ${ }^{16-18,21-27}$

\begin{tabular}{|c|c|c|}
\hline \multicolumn{2}{|c|}{ Médicaments Oculaires } & Médicaments Systémiques \\
\hline Anesthésiques topiques & $\begin{array}{l}\text { Proparacaïne }{ }^{17} \\
\text { Tétracaïne }{ }^{17} \\
\text { Lidocaïne }^{17} \\
\text { Benoxinate }^{17} \\
\text { AINS }^{17}\end{array}$ & Pilules contraceptives ${ }^{23}$ \\
\hline Cycloplégiques & Homatropine, atropine $\mathrm{e}^{17,18}$ & Hormonothérapie ${ }^{23}$ \\
\hline $\begin{array}{l}\text { Médicaments contre le } \\
\text { glaucome }\end{array}$ & $\begin{array}{l}\text { Apraclonidine }^{17} \\
\text { Brimonidine }^{17} \\
\text { Dorzolamide }^{17} \\
\text { Analogues des } \\
\text { prostaglandine }^{17,18} \\
\text { Dipivalyle épinéphrine }^{18} \\
\text { Échothiophate }^{18} \\
\text { Pilocarpine }^{18}\end{array}$ & Antidépesseurs ${ }^{23}$ \\
\hline Antiviraux & $\begin{array}{l}\text { Idoxuridine }^{18} \\
\text { Vidaribine }^{18} \\
\text { Trifluoridine }^{18} \\
\end{array}$ & AINS (kétoprofène; diclofénac) 23,25 \\
\hline Aminosides & $\begin{array}{l}\text { Tobramycine }^{18} \\
\text { Gentamycine }^{18} \\
\text { Néomycine }^{18} \\
\text { Vancomycine }^{18}\end{array}$ & Mésylate d'imatinib (Gleevec) ${ }^{24}$ \\
\hline Larmes artificielles & $\begin{array}{l}\text { Larmes artificielles à base de } \\
\text { lanoline }^{16}\end{array}$ & \\
\hline $\begin{array}{l}\text { Agents de conservation } \\
\text { des médicaments } \\
\text { ophtalmiques }\end{array}$ & $\begin{array}{l}\text { Chlorure de benzalkonium; } \\
\text { nitrate phénylmercurique } \\
17,18,22\end{array}$ & (inhibiteurs calciques comme l'amlodipine) \\
\hline
\end{tabular}

Tableau 3 : Signes oculaires de medicamentosa ${ }^{16,17,21,28}$

\begin{tabular}{|c|c|c|}
\hline Paupière & Conjonctive & Cornée \\
\hline Sténose ponctuelle ${ }^{21,28}$ & Réaction folliculaire ou papillaire ${ }^{21,28}$ & Épithéliopathie ponctuée superficielle ${ }^{21,28}$ \\
\hline \multirow{6}{*}{ Edème oculaire et/ou périorbital ${ }^{17,21}$} & Injection bulbaire $^{1,21}$ & Trouble épithélial, ulcération ${ }^{16,21}$ \\
\hline & $\begin{array}{l}\text { Amenuisement du symblépharon et/ou } \\
\text { du fornix }{ }^{1,21}\end{array}$ & Ulcérationl6, ${ }^{28}$ \\
\hline & \multirow{4}{*}{ Scarification ${ }^{21,28}$} & Scarification $^{16}$ \\
\hline & & Pannus $^{16}$ \\
\hline & & Néovascularisation ${ }^{16}$ \\
\hline & & Perforation $^{16}$ \\
\hline
\end{tabular}


Tableau 4a : Chimiose conjonctivale avec étiologie systémique d’hypersensibilité/d'allergie

\begin{tabular}{|c|c|c|c|c|}
\hline Association & Présentation & Symptomatologie & Bilan & $\begin{array}{l}\text { Coordination du } \\
\text { Spécialiste de la Santé }\end{array}$ \\
\hline Aliment & \multirow[t]{2}{*}{ Aiguë } & $\begin{array}{l}\text { Démangeaisons cutanées, bouffées } \\
\text { vasomotrices, œdème de l'œil, des } \\
\text { lèvres, de la langue et de la luette } \\
\text { (90,9\%), urticaire ( } 86,9 \%) \text {, symptômes } \\
\text { cardiovasculaires comme la syncope, } \\
\text { l'hypotension, l'incontinence urinaire, } \\
\text { les douleurs thoraciques ( } 31,3 \%) \text {, } \\
\text { l'hypotension ( } 26,3 \%) \text {, les symptômes } \\
\text { respiratoires comme la respiration } \\
\text { sifflante, le stridor, l'hypoxémie ( } 49,5 \%) \text {, } \\
\text { la dyspnée (47,5 \%), les symptômes } \\
\text { gastro-intestinaux comme les douleurs et } \\
\text { crampes abdominales, les vomissements, } \\
\text { la diarrhée (24,2 \%), l'œdème } \\
\text { angioneurotique (23,2\%), le prurit } \\
(27,3 \%) \text { et les symptômes neurologiques } \\
(20,2 \%)^{33} \text {. }\end{array}$ & \multirow[t]{2}{*}{$\begin{array}{l}\text { Test de la piqûre } \\
\text { cutanée et tests } \\
\text { de détection } \\
\text { sérique des } \\
\text { IgE spécifiques } \\
\text { d'allergènes (test } \\
\text { à allergosorbant } \\
\text { multiple, MAST), } \\
\text { hémogramme } \\
\text { avec analyse } \\
\text { différentielle, } \\
\text { IgE sériques } \\
\text { totales, protéine } \\
\text { cationique de } \\
\text { l'éosinophile, } \\
\text { série de tests } \\
\text { de la fonction } \\
\text { hépatique }^{33}\end{array}$} & \multirow[t]{2}{*}{$\begin{array}{l}\text { Allergologue ou service } \\
\text { des urgences selon la } \\
\text { gravité }\end{array}$} \\
\hline Médicament & & $\begin{array}{l}\text { Démangeaisons cutanées, bouffées } \\
\text { vasomotrices, œdème de l'œil, des } \\
\text { lèvres, de la langue et de la luette } \\
\text { ( } 69,4 \%) \text {, urticaire ( } 66,7 \%) \text {, symptômes } \\
\text { cardiovasculaires comme la syncope, } \\
\text { l'hypotension, l'incontinence urinaire, } \\
\text { les douleurs thoraciques ( } 63,9 \%) \text {, } \\
\text { l'hypotension (41,7 \%), les symptômes } \\
\text { respiratoires comme la respiration } \\
\text { sifflante, le stridor, l'hypoxémie ( } 45,8 \%) \text {, } \\
\text { la dyspnée ( } 45,8 \%) \text {, les symptômes } \\
\text { gastro-intestinaux comme les douleurs et } \\
\text { crampes abdominales, les vomissements, } \\
\text { la diarrhée ( } 25 \%), \text { l'œè̀me } \\
\text { angioneurotique (18,1 \%), le prurit } \\
(23 \%) \text { et les symptômes neurologiques } \\
(26,4 \%)^{33} \text {. }\end{array}$ & & \\
\hline
\end{tabular}

Tableau 4b : Chimiose conjonctivale avec étiologie systémique du syndrome des sinus caverneux

\begin{tabular}{|c|c|c|c|c|}
\hline & Présentation & Symptomatologie & Bilan & $\begin{array}{l}\text { Coordination du } \\
\text { Spécialiste de la Santé }\end{array}$ \\
\hline $\begin{array}{l}\text { Fistule } \\
\text { carotido- } \\
\text { caverneuse }\end{array}$ & $\begin{array}{l}\text { Subaiguë ou } \\
\text { chronique }\end{array}$ & $\begin{array}{l}\text { L'artérialisation en tire-bouchon } \\
\text { des veines conjonctivales } \\
\text { ( } 93 \%) \text {, l'exophtalmie ( } 84 \%) \text {, la } \\
\text { diminution de l'acuité visuelle } \\
\text { ( } 43 \%) \text {, la chimiose ( } 42 \%) \text {, } \\
\text { ophthalmoparésie ( } 52 \%: 73 \% \\
\text { de l'abducteur, } 5 \% \text { oculomoteur, } \\
22 \% \text { multiple) et bruit oculaire } \\
(28 \%)^{30}\end{array}$ & $\begin{array}{l}\text { Tomodensitométrie / } \\
\text { angiographie par } \\
\text { tomodensitométrie, ou } \\
\text { imagerie par résonance } \\
\text { magnétique / angiographie } \\
\text { par résonance magnétique, } \\
\text { ou densité des vaisseaux } \\
\text { capillaires péripapillaires } \\
(\mathrm{PCVD})^{47}\end{array}$ & Service des urgences \\
\hline $\begin{array}{l}\text { Thrombose } \\
\text { carotido- } \\
\text { caverneuse }\end{array}$ & Aiguë & $\begin{array}{l}\text { Fièvre, exophtalmie, ptose, } \\
\text { paralysie des nerfs crâniens } \\
\text { III, IV ou VI ( } 80-100 \%), \\
\text { œdème périorbital, maux de } \\
\text { tête, léthargie, troubles de la } \\
\text { conscience }(50-80 \%) \text {, œdème } \\
\text { périorbital et engorgement } \\
\text { veineux }{ }^{32}\end{array}$ & $\begin{array}{l}\text { Tomodensitométrie / } \\
\text { angiographie par } \\
\text { tomodensitométrie ou } \\
\text { imagerie par résonance } \\
\text { magnétique / angiographie } \\
\text { par résonance } \\
\text { magnétique }^{58}\end{array}$ & Service des urgences \\
\hline
\end{tabular}


Tableau 4c : Chimiose conjonctivale avec étiologie systémique de trouble thyroïdien (hyperthyrö̈die 1)

\begin{tabular}{|c|c|c|c|}
\hline Présentation & Symptomatologie & Bilan & $\begin{array}{l}\text { Coordination du } \\
\text { Spécialiste de la Santé }\end{array}$ \\
\hline \multirow[t]{3}{*}{$\begin{array}{l}\text { Sous-aiguë/ } \\
\text { chronique }\end{array}$} & $\begin{array}{l}\text { Patients plus âgés (> } 70 \text { ans })^{34} \\
\text { Tachycardie }(71 \%) \text {, fatigue }(56 \%) \text {, perte pondérale } \\
(50 \%) \text {, tremblements }(44 \%) \text {, dyspnée }(41 \%) \text {, apathie } \\
(41 \%) \text {, anorexie }(32 \%) \text {, nervosité }(31 \%) \text {, réflexes } \\
\text { hyperactifs }(28 \%), \text { faiblesse }(27 \%) \text {, dépression }(24 \%) \\
\text { et sudation accrue }(24 \%)^{34} \text {, alopécie }{ }^{31} \text { et myxœème ne } \\
\text { retenant pas le godet }{ }^{31,52}\end{array}$ & \multirow{3}{*}{$\begin{array}{l}\text { Test de la piqûre } \\
\text { cutanée et tests de } \\
\text { détection sérique } \\
\text { des IgE spécifiques } \\
\text { d'allergènes (test } \\
\text { à allergosorbant } \\
\text { multiple, MAST), } \\
\text { hémogramme } \\
\text { avec analyse } \\
\text { différentielle, IgE } \\
\text { sériques totales, } \\
\text { protéine cationique } \\
\text { de l'éosinophile, } \\
\text { série de tests de la } \\
\text { fonction hépatique }\end{array}$} & \multirow[t]{3}{*}{$\begin{array}{l}\text { Allergologue ou service } \\
\text { des urgences selon la } \\
\text { gravité }\end{array}$} \\
\hline & $\begin{array}{l}\text { Patients plus jeunes }(<50 \text { ans })^{34} \\
\text { Tachycardie }(96 \%) \text {, réflexes hyperactifs }(96 \%) \text {, sudation } \\
\text { accrue }(95 \%) \text {, intolérance à la chaleur }(92 \%) \text {, fatigue } \\
(84 \%) \text {, nervosité }(84 \%) \text {, tremblement }(84 \%) \text {, polydipsie } \\
(67 \%) \text {, faiblesse }(61 \%) \text {, augmentation de l'appétit } \\
(57 \%) \text {, dyspnée }(56 \%) \text {, perte pondérale }(51 \%) \text {, diarrhée } \\
(43 \%) \text {, anorexie }(32 \%) \text {, apathie }(25 \%) \text { et dépression } \\
(22 \%)^{34} \text {, alopécie }{ }^{31} \text {, œdème prétibial ne retenant pas le } \\
\text { godet }(1-5 \%)^{52}\end{array}$ & & \\
\hline & 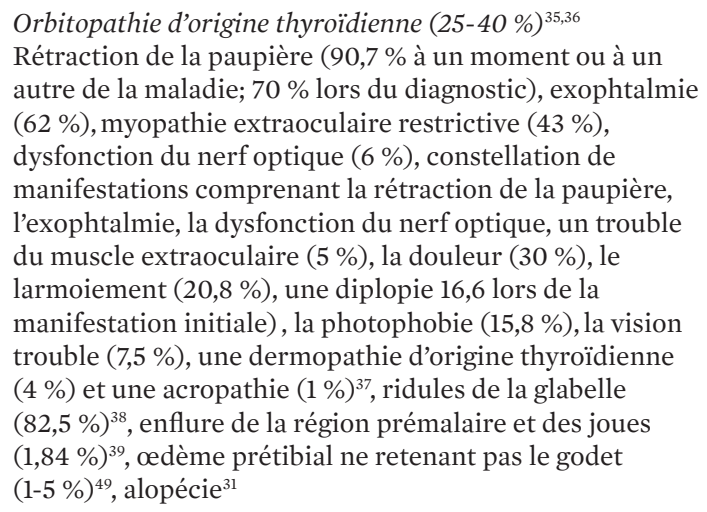 & & \\
\hline
\end{tabular}


Tableau 4d: Chimiose conjonctivale avec étiologie systémique du syndrome de la veine cave supérieure

\begin{tabular}{|c|c|c|c|c|}
\hline & Présentation & Symptomatologie & Bilan & $\begin{array}{l}\text { Coordination du } \\
\text { Spécialiste de la Santé }\end{array}$ \\
\hline $\begin{array}{l}\text { Causes malignes }(60 \%) 40 \\
\text { Lymphome }(8 \%)^{40} \\
\text { Carcinome bronchogène }(46 \%)^{40} \\
\text { - Cancer du poumon à petites } \\
\text { cellules }(22 \%)^{40} \\
\text { - Cancer du poumon non à } \\
\text { petites cellules }(24 \%)^{40} \\
\text { Tumeur des cellules germinales } \\
(8 \%)^{40} \\
\text { - Cancer métastatique de la } \\
\text { prostate }(1,2 \%)^{40} \\
\text { - Cancer du thymus }(1,2 \%)^{40} \\
\text { - Adénocarcinome d'un site } \\
\text { inconnu }(1,2 \%)^{40}\end{array}$ & $\begin{array}{l}\text { Sous-aiguë/ } \\
\text { chronique }\end{array}$ & \multirow[t]{6}{*}{$\begin{array}{l}\text { Symptômes généralisés } \\
\text { du SVC : Enflure du } \\
\text { visage ou de la nuque } \\
\text { ( } 82 \%) \text {, enflure des } \\
\text { extrémités des membres } \\
\text { supérieurs ( } 68 \% \text { ), } \\
\text { dyspnée ( } 66 \%) \text {, toux } \\
(50 \%) \text { et dilatation } \\
\text { des veines collatérales } \\
\text { thoraciques ( } 38 \%)^{40} \text {, } \\
\text { jaunisse dans le } \\
\text { lymphome et syphilis }{ }^{51}\end{array}$} & \multirow[t]{6}{*}{$\begin{array}{l}\text { TDM ou IRM } \\
\text { thoracique à } \\
\text { l'évaluation } \\
\text { initiale et } \\
\text { biopsie } \\
\text { tissulaire des }^{\text {masses }}\end{array}$} & \multirow[t]{2}{*}{ Oncologue } \\
\hline & \multirow[t]{5}{*}{$\begin{array}{l}\text { Sous-aiguë/ } \\
\text { chronique }\end{array}$} & & & \\
\hline \multirow{2}{*}{$\begin{array}{l}\text { Appareil intravasculaire (71 \%), } \\
\text { cathéters de dialyse ( } 5 \%) \text {, fil de } \\
\text { stimulateur cardiaque }(1,2 \%) \text { et } \\
\text { cathéter de Hickman }(1,2 \%)^{40}\end{array}$} & & & & Cardiologue \\
\hline & & & & $\begin{array}{l}\text { Maladie infectieuse; } \\
\text { cardiologue }\end{array}$ \\
\hline $\begin{array}{l}\text { Médastinite fibreuse }(8 \%)^{40} \\
\text { - Lymphome, histoplasmose, } \\
\text { tuberculose et anévrisme de } \\
\text { l'aorte syphilitique }{ }^{40}\end{array}$ & & & & Cardiologue \\
\hline $\begin{array}{l}\text { Autres }(3,6 \%)^{40} \\
\text { - Traitement de l'hématome } \\
\text { après une dissection aortique; } \\
\text { pseudotumeur; thrombose } \\
\text { primaire de la } \mathrm{VCS}^{40}\end{array}$ & & & & \\
\hline
\end{tabular}


Tableau 4e : Chimiose conjonctivale avec étiologie systémique d'hypervolémie

\begin{tabular}{|c|c|c|c|c|}
\hline & Présentation & Symptomatologie & Bilan & $\begin{array}{l}\text { Coordination du } \\
\text { Spécialiste de la Santé }\end{array}$ \\
\hline $\begin{array}{l}\text { Défaillance } \\
\text { cardiorespiratoire }\end{array}$ & $\begin{array}{l}\text { Sous-aiguë/ } \\
\text { chronique }\end{array}$ & $\begin{array}{l}\text { Toutes les formes de } \\
\text { dyspnée (87-93\%), de } \\
\text { dyspnée d'effort (86-97 \%), } \\
\text { d'orthopnée (10-59\%), } \\
\text { de dyspnée paroxysmale } \\
\text { (13-39\%), de dyspnée } \\
\text { au repos (1-6\%), de gain } \\
\text { pondéral(5-15\%), de } \\
\text { distension de la veine } \\
\text { jugulaire (5-54\%), de } \\
\text { réflexe jugulaire abdominal } \\
\text { (6\%), d'œedème (35-70\%), } \\
\text { de bruit de galop du cœur } \\
\text { (1-26\%), de râles (25-45\%) } \\
\text { et d'ascite (3-17\%) } \\
\text { Jaunisse en cas de } \\
\text { défaillance cardio-rénale }\end{array}$ & $\begin{array}{l}\text { Hématocrite, taux } \\
\text { de protéines, taux } \\
\text { d'albumine, taux } \\
\text { de créatinine, taux } \\
\text { d'azote uréique dans } \\
\text { le sang, osmolité } \\
\text { plasmatique et } \\
\text { densité de l'urine, } \\
\text { radiographie } \\
\text { thoracique, } \\
\text { cathétérisation de } \\
\text { l'artère pulmonaire, } \\
\text { PRO BNP, tests } \\
\text { cardiaques, } \\
\text { échocardiographie } \\
\text { transthoracique }\end{array}$ & $\begin{array}{l}\text { Cardiologue; service } \\
\text { des urgences }\end{array}$ \\
\hline \multirow{3}{*}{ 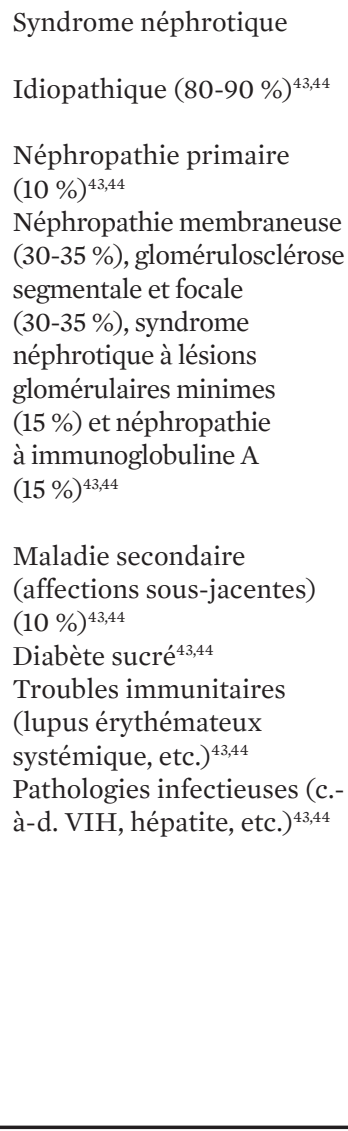 } & \multirow[t]{3}{*}{$\begin{array}{l}\text { Sous-aiguë/ } \\
\text { chronique }\end{array}$} & $\begin{array}{l}\text { Symptômes généraux } \\
\text { de maladie primaire ou } \\
\text { secondaire : Malaises, } \\
\text { gain pondéral, urine } \\
\text { spumeuse, œdème } \\
\text { périorbital du matin, } \\
\text { ongles blanchâtres avec } \\
\text { ou sans bandes blanches } \\
\text { (lignes de Muehrcke), } \\
\text { xanthomes cutanés et } \\
\text { alopécie }{ }^{42} \\
\text { Edème périphérique } \\
\text { retenant le godet marqué } \\
\text { en soirée } \\
\text { Jaunisse }^{51}\end{array}$ & \multirow{3}{*}{$\begin{array}{l}\text { Analyse d'urine } \\
\text { avec rapport } \\
\text { protéines/créatinine, } \\
\text { hémogramme et } \\
\text { tests de coagulation, } \\
\text { électrolytes pour } \\
\text { vérifier la fonction } \\
\text { rénale, tests de la } \\
\text { fonction hépatique, } \\
\text { glucose et HbAlC, } \\
\text { tests ciblés pour les } \\
\text { troubles décelés avec } \\
\text { les antécédents et } \\
\text { l'examen physique } \\
\text { anticorps } \\
\text { antinucléaires, } \\
\text { anticorps anti-ADN } \\
\text { bicaténaires, anticorps } \\
\text { anti-Sm, anticorps des } \\
\text { anti-phospholipides, } \\
\text { anticorps anti-ARN }{ }^{42,43}\end{array}$} & \multirow[t]{3}{*}{$\begin{array}{l}\text { Néphrologue; } \\
\text { rhumatologue }\end{array}$} \\
\hline & & $\begin{array}{l}\text { Perte de poids, polyurie, } \\
\text { polydipsie, polyphagie }\end{array}$ & & \\
\hline & & $\begin{array}{l}\text { Lupus érythémateux } \\
\text { systémique : Arthrite } \\
\text { et/ou arthralgie ( } 86 \%) \text {, } \\
\text { éruptions cutanées } \\
\text { ( } 61 \%) \text {, anémie }(55 \%) \text {, } \\
\text { photosensibilité ( } 48 \%) \text {, } \\
\text { fièvre ( } 43 \%) \text {, ulcère de } \\
\text { la bouche }(43 \%) \text {, maux } \\
\text { de tête }(36 \%) \text {, fatigue, } \\
\text { malaise et faiblesse (35\%) } \\
\text { et alopécie ( } 35 \%)^{45} \text {. } \\
\text { Edème généralisé ne } \\
\text { retenant pas le godet }{ }^{18} \\
\text { Edème déclive retenant } \\
\text { le godet à la fin du jour }{ }^{18}\end{array}$ & & \\
\hline Néphropathie & $\begin{array}{l}\text { Sous-aiguë/ } \\
\text { chronique }\end{array}$ & 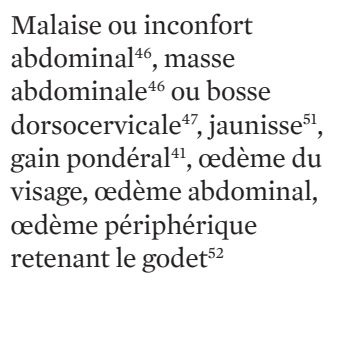 & $\begin{array}{l}\text { Tests de la fonction } \\
\text { rénale (inclure l'AST, } \\
\text { l'ALT, le rapport AST/ } \\
\text { ALT, la phosphatase } \\
\text { alcaline et la } \\
\gamma \text {-glutamyltransférase, } \\
\text { la bilirubine, } \\
\text { l'albumine, DFG), } \\
\text { échographie hépatique } \\
\text { ou IRM }\end{array}$ & Hépatologue \\
\hline
\end{tabular}


Tableau 5 : Mécanisme d'action des troubles systémiques accompagnant la chimiose conjonctivale $2,6,18,33,40,42,49,53-58$

\begin{tabular}{|c|c|c|c|}
\hline \multicolumn{3}{|c|}{ Étiologie } & Mécanisme d'action Systémique \\
\hline \multirow{2}{*}{$\begin{array}{l}\text { Atteintes } \\
\text { du sinus } \\
\text { caverneux }\end{array}$} & \multicolumn{2}{|c|}{ Fistule carotido-caverneuse } & $\begin{array}{l}\text { Altération du drainage veineux secondaire à une communication } \\
\text { anormale entre l'artère carotide interne et le sinus caverneux ou } \\
\text { les ramifications vers les méninges de l'artère carotide interne ou } \\
\text { de l'artère carotide externe et des sinus caverneux }{ }^{57}\end{array}$ \\
\hline & \multicolumn{2}{|c|}{ Thrombose carotido-caverneuse } & $\begin{array}{l}\text { Altération du drainage veineux secondaire à une infection du nez, } \\
\text { des sinus, des oreilles ou des dents } \mathrm{s}^{32,58}\end{array}$ \\
\hline \multicolumn{3}{|c|}{ Hypersensibilité/allergie } & $\begin{array}{l}\text { Réaction allergique aux squames animales, au pollen, aux aliments, } \\
\text { aux médicaments, au venin ou aux drogues induite par la libération } \\
\text { d'histamine, de sérotonine et de bradykinine, accompagnée d'une } \\
\text { migration des cellules polymorphonucléaires, laquelle provoque la } \\
\text { dilatation des artérioles, la hausse du gradient de pression entre les } \\
\text { capillaires artériolaires et les veinules ainsi que l'extravasation du } \\
\text { liquide intravasculaire et œedème de vasodilatation consécutif }{ }^{, 18,33}\end{array}$ \\
\hline \multicolumn{3}{|l|}{ Hyperthyroïdie } & $\begin{array}{l}\text { Accumulation de glycosaminoglycanes dans les tissus } \\
\text { conjonctifs, entraînant un œèeme et une inflammation des } \\
\text { muscles extraoculaires, du tissu conjonctif orbital et adipeux, } \\
\text { l'augmentation du volume orbital et la diminution du drainage } \\
\text { veineux et lymphatique }{ }^{49,53}\end{array}$ \\
\hline \multirow{6}{*}{$\begin{array}{l}\text { Syndrome de } \\
\text { la veine cave } \\
\text { supérieure }\end{array}$} & \multicolumn{2}{|l|}{ Lymphome } & $\begin{array}{l}\text { Ganglion lymphatique hypertrophié comprimant la veine } \\
\text { supérieure et causant ainsi une diminution du drainage veineux }{ }^{40}\end{array}$ \\
\hline & \multicolumn{2}{|c|}{$\begin{array}{l}\text { Bronchogène. Carcinome (cancer du } \\
\text { poumon à petites cellules ou non) }\end{array}$} & $\begin{array}{l}\text { Compression massive de la veine cave supérieure causant une } \\
\text { diminution du drainage veineux }{ }^{40}\end{array}$ \\
\hline & \multicolumn{2}{|c|}{$\begin{array}{l}\text { Tumeur des cellules germinales } \\
\text { (prostate, thymus, adénocarcinome) }\end{array}$} & $\begin{array}{l}\text { Compression massive de la veine cave supérieure causant une } \\
\text { diminution du drainage veineux }{ }^{40}\end{array}$ \\
\hline & \multicolumn{2}{|c|}{ Dispositifs intravasculaires } & $\begin{array}{l}\text { Dispositif qui comprime la veine cave supérieure causant une } \\
\text { diminution du drainage veineux }{ }^{40}\end{array}$ \\
\hline & \multicolumn{2}{|c|}{$\begin{array}{l}\text { Médastinite fibreuse (lymphome; } \\
\text { histoplasmose; tuberculose; anévrisme } \\
\text { de l'aorte d'origine syphilitique) }\end{array}$} & $\begin{array}{l}\text { Cicatrisation fibreuse des tissus qui comprime la veine cave } \\
\text { supérieure causant une diminution du drainage veineux }{ }^{40}\end{array}$ \\
\hline & \multicolumn{2}{|c|}{$\begin{array}{l}\text { Autre (hématome après réparation par } \\
\text { dissection aortique; pseudotumeur; } \\
\text { thrombose primaire de la VCS) }\end{array}$} & $\begin{array}{l}\text { Compression de la veine cave supérieure causant une diminution } \\
\text { du drainage veineux }{ }^{40}\end{array}$ \\
\hline \multirow{4}{*}{ Hypervolémie } & \multicolumn{2}{|c|}{ Défaillance cardiorespiratoire } & $\begin{array}{l}\text { Dilatation du système vasculaire oculaire causée par l'hypoxie, } \\
\text { l'hypercapnie, qui entrave le retour veineux thoracique en raison } \\
\text { d'une ventilation sous pression positive qui induit une distension } \\
\text { veineuse jugulaire et une surcharge de liquides }\end{array}$ \\
\hline & \multirow[b]{2}{*}{$\begin{array}{l}\text { Syndrome } \\
\text { néphrotique }\end{array}$} & $\begin{array}{l}\text { Néphropathie } \\
\text { primaire }\end{array}$ & $\begin{array}{l}\text { Faible taux de protéines dans le sang (hypoprotéinémie) causant } \\
\text { une diminution de la pression osmotique colloïdale, laquelle } \\
\text { altère le gradient osmotique entre le plasma et le liquide } \\
\text { interstitiel, causant ainsi la rétention du sel et de l'eau ainsi que } \\
\text { l'évacuation des liquides dans l'espace extracellulaire }{ }^{6,42,54}\end{array}$ \\
\hline & & $\begin{array}{l}\text { Néphropathie } \\
\text { secondaire, } \\
\text { en particulier } \\
\text { le lupus } \\
\text { érythémateux } \\
\text { systémique }\end{array}$ & $\begin{array}{l}\text { Patients présentant des signes d'affection systémique : Faible } \\
\text { taux de protéines dans le sang (hypoprotéinémie) causant une } \\
\text { diminution de la pression osmotique colloïdale, laquelle altère le } \\
\text { gradient osmotique entre le plasma et le liquide interstitiel, causant } \\
\text { ainsi l'évacuation du liquide dans l'espace extracellulaire } \\
\text { Patients ne présentant aucun signe d'affection systémique : } \\
\text { Edème localisé à partir de complexes immunitaires, causant une } \\
\text { augmentation de la présence de cellules polymorphonucléaires et } \\
\text { une augmentation de l'œème et de l'inflammation }{ }^{54,55}\end{array}$ \\
\hline & \multicolumn{2}{|c|}{ Affections hépatiques } & $\begin{array}{l}\text { Faible taux d'albumine dans le sang (hypoalbuminémie) causant } \\
\text { une diminution de la pression osmotique colloïdale, une } \\
\text { augmentation de la pression oncotique et l'évacuation des liquides } \\
\text { dans les tissus mous oculaires }\end{array}$ \\
\hline
\end{tabular}


Table 6: Cutaneous presentations in systemic diseases that may present with conjunctival chemosis ${ }^{18,31,33,42,45,48,49,51,52,68-70}$

\begin{tabular}{|c|c|c|c|c|c|c|}
\hline \multirow{2}{*}{\multicolumn{2}{|c|}{ Trouble Systémique }} & \multicolumn{5}{|c|}{ Présentation Cutanée } \\
\hline & & Rash & $\begin{array}{c}\text { Alopecia } \\
\text { (Yes Or No) }\end{array}$ & $\begin{array}{c}\text { Jaundice } \\
\text { (Yes Or No) }\end{array}$ & \multicolumn{2}{|c|}{$\begin{array}{l}\text { Edema (None; Pitting; } \\
\text { Non-Pitting) }\end{array}$} \\
\hline \multirow[t]{2}{*}{$\begin{array}{l}\text { Hypersensibilité/ } \\
\text { allergie }\end{array}$} & $\begin{array}{l}\text { D’origine } \\
\text { médicamenteuse }\end{array}$ & $\begin{array}{l}\text { Urticaire (bosses rougeâtres ou } \\
\text { plaques pâles accompagnées } \\
\text { d'œedème) } 66,7 \%^{33}\end{array}$ & Non & Non & \multicolumn{2}{|l|}{ Aucun(e) } \\
\hline & D'origine alimentaire & $\begin{array}{l}\text { Urticaire (bosses rougeâtres ou } \\
\text { plaques pâles accompagnées } \\
\text { d'œdème; } 86,9 \% \%^{33}\end{array}$ & Non & Non & \multicolumn{2}{|l|}{$\operatorname{Aucun}(\mathrm{e})$} \\
\hline \multicolumn{2}{|c|}{ Hyperthyroïdie/maladie de Graves } & $\begin{array}{l}\text { Myxœedème prétibial se } \\
\text { présentant avec un érythème } \\
\text { asymptomatique chaud, } \\
\text { humide, doux, velouté et lisse } \\
\text { présentant une éléphantiasis } \\
\text { prononcée }(1-5 \%)^{4,52}\end{array}$ & Oui $^{10}$ & Non & \multicolumn{2}{|c|}{$\begin{array}{l}\text { Myxœdème ne retenant } \\
\text { pas le godet }{ }^{31,52}\end{array}$} \\
\hline $\begin{array}{l}\text { Syndrome de la veine } \\
\text { cave supérieure }\end{array}$ & $\begin{array}{l}\text { Tumeur, ganglion } \\
\text { lymphatique, } \\
\text { dommages } \\
\text { aux vaisseaux } \\
\text { lymphatiques, } \\
\text { radiothérapie }^{51} \\
\end{array}$ & Non & Non & $\begin{array}{l}\text { Yes, if } \\
\text { lymphoma51 }\end{array}$ & \multicolumn{2}{|c|}{ Ne gardant pas le godet ${ }^{52}$} \\
\hline \multirow[t]{3}{*}{$\begin{array}{l}\text { Syphilis } \\
\text { (se manifestant dans les } \\
\text { zones extragénitales) }\end{array}$} & Primaire & $\begin{array}{l}\text { Chancre without basal } \\
\text { induration with edges rising } \\
\text { above the surrounding } \\
\text { surface }(2 \%)^{69}\end{array}$ & \multirow[t]{3}{*}{ Yes } & \multirow[t]{3}{*}{ Yes $^{51}$} & \multirow{2}{*}{\multicolumn{2}{|c|}{$\operatorname{Aucun}(\mathrm{e})$}} \\
\hline & Secondaire & $\begin{array}{l}\text { Maculaire, populaire, } \\
\text { papulosquameux, lésion } \\
\text { pustulaire ( } 4,76 \% \text { à } 59 \%) \text {, } \\
\text { selon l'emplacement, avec } \\
\text { une présentation plus élevée } \\
\text { sur les semelles, le tronc, les } \\
\text { bras, les paumes, les jambes }{ }^{70}\end{array}$ & & & & \\
\hline & Tertiaire & $\begin{array}{l}\text { Lésions nécrotiques centrées } \\
\text { formant une gomme }(15 \%)^{48}\end{array}$ & & & \multicolumn{2}{|c|}{$\begin{array}{l}\text { Retenant le godet, } \\
\text { si le patient souffre } \\
\text { d'insuffisance cardiaque }^{52}\end{array}$} \\
\hline \multirow[t]{4}{*}{ Hypervolémie } & $\begin{array}{l}\text { Insuffisance } \\
\text { cardiaque congestive }\end{array}$ & Non & Non & $\begin{array}{l}\text { Oui, en } \\
\text { présence } \\
\text { d'insuffisance } \\
\text { cardiaque et } \\
\text { rénale }^{51}\end{array}$ & Retenant & godet $^{51}$ \\
\hline & \multirow[t]{2}{*}{$\begin{array}{l}\text { Syndrome } \\
\text { néphrotique }\end{array}$} & Néphropathie primaire & $\begin{array}{l}\text { Ongles blanchâtres } \\
\text { avec ou sans bandes } \\
\text { blanches (lignes } \\
\text { de Muehrcke) } \\
\text { et xanthomes } \\
\text { cutanés }^{42}\end{array}$ & \multirow[t]{2}{*}{ Oui $^{48}$} & \multirow[t]{2}{*}{ Oui $^{51}$} & \multirow{2}{*}{$\begin{array}{l}\text { Edème } \\
\text { généralisé ne } \\
\text { retenant pas } \\
\text { le godet } 18,52 \\
\text { ou œedème } \\
\text { déclive } \\
\text { retenant le } \\
\text { godet à la fin } \\
\text { du jour }{ }^{18}\end{array}$} \\
\hline & & $\begin{array}{l}\text { Lupus érythémateux } \\
\text { systémique }\end{array}$ & $\begin{array}{l}\text { Ongles blanchâtres } \\
\text { avec ou sans bandes } \\
\text { blanches (lignes } \\
\text { de Muehrcke) } \\
\text { et xanthomes } \\
\text { cutanés }^{42} \\
\text { Toute forme } \\
\text { d'éruption cutanée } \\
\text { (> } 80 \%)^{68} \\
\text { Éruption en forme } \\
\text { de papillon }(61 \%)^{45}\end{array}$ & & & \\
\hline & Néphropathie & & Non & Oui $^{51}$ & $\begin{array}{l}\text { Retenant } \\
\text { le godet }{ }^{52}\end{array}$ & \\
\hline
\end{tabular}




\section{RÉFÉRENCES}

1. Hunter PA. The conjunctiva: diseases and tumours. In: Spalton DJ, Hitchings RA, Hunter PA, eds. Atlas of Clinical Ophthalmology, 2nd edn. London: , 1998:3-5.

2. Kanski JJ, Bowling B. Clinical CITY OF PUBLICATION: PUBLISHER, YEAR OF PUBLICATION:131-66.

3. Dutton J. Atlas of Clinical and Surgical Orbital Anatomy, 2nd edn. CITY OF PUBLICATION: PUBLISHER, 2011:129-64.

4. Remington L. Clinical Anatomy of the Visual System. Boston: Butterworth-Heinemann, 1998:137-56.

5. Minckler MR, Newell C, Drummond B. Chemosis from trauma. West J Emerg Med 2014;15(4):357-8.

6. Kalin NS, Orlin SE, Wulc AE, et al. Chronic localized conjunctival chemosis. Cornea 1996; 15(3):295-300.

7. Weinfeld AB, Burke R, Codner MA. The comprehensive management of chemosis following cosmetic lower blepharoplasty. Plast Reconstr Surg 2008;122:579-86.

8. Kim HK, Kim WS. Chronic unilateral chemosis following the use of amlodipine besylate. BMC Ophthalmol 2014;14:124.

9. Gans H, Sekula J, Wlodyka J. Treatment of acute orbital complications. Arch Otolaryngol 1974;100:329-32.

10. Cioffi GA, Van Buskirk EM. Clinical manifestations of the glaucomas. In: Wright KW, ed. Textbook of Ophthalmology. Baltimore, MD: , 1997:597-624

11. Medeiros LJ, Harmon DJ, Lingood RM, Harris NL. Immunohistologic features predict clinical behavior of orbital and conjunctival lymphoid infiltrates. Blood 1989; 74:2121-9.

12. Schroder K, Finis D, Meller S, Buhren BA, Wagenmann M, Geerling G. Seasonal and perennial allergic rhinoconjunctivitis. Laryngorhinootologie 2017; 96(2):89-97.

13. Allegrini D, Reposi S, Nocerino E, Pece A. Odontogenic orbital cellulitis associated with cavernous sinus thrombosis and pulmonary embolism: a case report. J Med Case Rep 2017 Jun 20;11(1):16414. Michael KB, Rotchford A, Ramaesh K. Conjunctival chemosis as a specific feature of pseudomonas aeruginosa corneal ulcers. Cornea 2016;35:1182-4.

15. Murthy SI, Sati A, Sangwan V. Infectious scleritis mimicking severe ocular inflammation: atypical initial presentation. BMJ Case Rep 2013 Feb 20;2013.

16. Graue-Hernández EO, Navas A, Ramírez-Miranda A. Toxic keratoconjunctivitis. In: Holland EJ, Mannis MJ, Lee WB, eds. Ocular Surface Disease: Cornea, Conjunctiva and Tear Film. London: W.B. Saunders, 2013:189-93.

17. Paley GL, Lubniewski AJ, Reidy JJ, Farooq AV. Toxic keratoconjunctivitis. Eye Contact Lens 2018 Sep;44 Suppl 1:S8-S15.18. Liesegang TJ. Conjunctiva. In: Wright KW, ed. Textbook of Ophthalmology. Baltimore, MD: Williams \& Wilkins, 1997:665-90. 19. Meyer, DR. Orbital fractures. In: Tasman W, Jaeger EA, eds. Duane' Foundations of Clinical Ophthalmology, 15th edn. Philadelphia: Lippincott Williams \& Wilkins, 2009: chap 48.

20. Mackool RJ, Monsanto VR. Role of the bandage contact lens in the management of concomitant keratoconjunctivitis medicamentosa and cystoid macular edema. J Cataract Refract Surg 2002;28:1714.

21. Wilson FM II. Adverse external ocular effects of topical ophthalmic medications. Surv Ophthalmol 1979;24:57-88.

22. Chen W, Li Z, Hu J, et al. Corneal alterations induced by topical application of benzalkonium chloride in rabbit. PLoS One 2011;6:e26103.

23. Fraunfelder FW. Corneal toxicity from topical ocular and systemic medications. Cornea 2006; 25:1133-8.

24. Jin J, Chen H, Cao L. Management of conjunctival chemosis secondary to imatinib treatment for chronic myelogenous leukemia. Leuk Res 2009;33: e18-e19.

25. Fuentes V, de Frutos C, de Barrio M, et al. Unilateral conjunctival chemosis as a unique symptom of nonsteroidal anti-inflammatory drug intolerance. J Investig Allergol Clin Immunol 2007;17(1): 62-4.

26. Kyeong HK, Kim WS. Chronic unilateral chemosis following the use of amlodipine besylate. BMC Ophthalmol 2014;14:124.

27. Makani $\mathrm{H}$, Bangalore $\mathrm{S}$, Romero J, Wever-Pinzon O, Messerli FH: Effect of renin-angiotensin system blockade on calcium channel blocker associated peripheral edema. Am J Med 2011;124(2):128-35.

28. Spalton DJ, Hitchings RA, Hunter PA. Atlas of Clinical Ophthalmology, 3rd edn. Oxford: Elsevier Mosby, 2004.
29. Krachmer JH, Mannis MJ, Holland EJ. Cornea: Fundamentals, Diagnosis, and Management, 3rd edn. New York: Elsevier Mosby, 2011.

30. Preechawat P, Narmkerd P, Jiarakongmun P, et al. Dural carotid cavernous sinus fistula: ocular characteristics, endovascular management and clinical outcome. J Med Assoc Thai 2008;91:852-8.

31. Ai J, Leonhardt JM, Heymann WR. Autoimmune thyroid diseases: etiology, pathogenesis, and dermatologic manifestations. J Am Acad Dermatol 2003;48:641-59.

32. Lemos J, Eggenberger E. Neuro-ophthalmological emergencies. Neurohospitalist 2015;5(4):223-33.

33. Kim SY, Kim MH, Cho YJ. Different clinical features of anaphylaxis according to cause and risk factors for severe reactions. Allergol Int 2018; 67(1):96-102.

34. Trivalle C, Doucet J, Chassagne P, et al. Differences in the signs and symptoms of hyperthyroidism in older and younger patients. J Am Geriatr Soc 1996 Jan;44(1):50-3.

35. Bartalena L, Pinchera A, Marcocci C. Management of Graves' ophthalmopathy: Reality and perspectives. Endocr Rev 2000;21:168-99.

36. Wiersinga WM, Bartalena L. Epidemiology and prevention of Graves' ophthalmopathy. Thyroid 2002;12:855-60.

37. Bartley GB, Fatourechi V, Kadrmas EF, et al. Clinical features of Graves' ophthalmopathy in an incidence cohort. Am J Ophthalmol 1996;121:284-90.

38. Saks ND, Burnstine MA, Putterman AM. Glabellar rhytids in thyroid-associated orbitopathy. Ophthalmic Plast Reconstr Surg 2001;17:91-5

39. Kim BJ, Kazim M. Prominent premalar and cheek swelling: a sign of thyroid associated orbitopathy. Ophthalmic Plast Reconstr Surg 2006;22:457-60

40. Rice TW, Rodriguez M, Light RW. The superior vena cava syndrome: clinical characteristics and evolving etiology. Medicine 2006;85:37-42.

41. Tuy T, Peacock WF. Fluid overload assessment and management in heart failure patients. Semin Nephrol 2012;32(1):112-20.

42. McCloskey O, Maxwell AP. Diagnosis and management of nephrotic syndrome. Practitioner 2017;261(1801):11-5

43. Hull RP, Goldsmith DJ. Nephrotic syndrome in adults. BMJ 2008;336(7654):1185-9.

44. Kodner C. Diagnosis and management of nephrotic syndrome in adults. Am Fam Physician 2016;93(6):479-85.

45. Ozbek S, Sert M, Paydas S, Soy M. Delay in the diagnosis of SLE: The importance of arthritis/arthralgia as the initial symptom Acta Med Okayama 2003;57(4):187-90.

46. Tomic D, Kemp WW, Roberts SK. Nonalcoholic fatty liver disease: current concepts, epidemiology and management strategies. Eur J Gastroenterol Hepatol 2018 Oct;30(10):1103-15.

47. Cheung O, Kapoor A, Puri P, et al. The impact of fat distribution on the severity of nonalcoholic fatty liver disease and metabolic syndrome. Hepatology 2007;46:1091-100.

48. Thomann KH. Syphilis. In: Marks ES, Adamczyk DT, Thomann KH, eds. Primary Eyecare in Systemic Disease. Norwalk, CT:Appleton \& Lange, 1995:404-16.

49. Fatourechi V. Pretibial myxedema: pathophysiology and treatment options. Am J Clin Dermatol 2005;6:295-309.50. Straka C, Ying J, Kong FM, Willey CD, Kaminski J, Kim DWN. Review of evolving etiologies, implications and treatment strategies for the superior vena cava syndrome. Springerplus 2016 Feb 29;5:229.51. Blendis LM. Jaundice in systemic disease. Baillieres Clin Gastroenterol 1989 Apr;3(2):431-45.52. Whiting E, McCready ME. Pitting and non-pitting edema. Med J Aust 2016; 205(4):157-8

53. Bahn RS, Heufelder AE. Mechanisms of disease: pathogenesis of Grave's ophthalmopathy. N Engl J Med 1993;329:1468-75.

54. Peponis V, Chalkiadakis S, Ergin S, Kyttaris VC. Chemosis as a presenting symptom of systemic lupus erythematosus. Lupus 2010 Jul;19(8):997-1001.55. Jankauskiene A, Buteikiene I. Severe chemosis in a patient with nephritic syndrome. Eur J Pediatr 2009;168(4):507-8

56. Glauser FL. Bilateral chemosis and conjunctival engorgement in cardiopulmonary failure. Chest 1974;66(4):389-94

57. Srinivasan A, Biro NG, Murchison AP, et al. Efficacy of orbital color doppler imaging and neuroimaging in the diagnosis of carotid cavernous fistulas. Ophthalmic Plast Reconstr Surg 2016;33(5):340-4. 
58. Al-Mufti F, Amuluru K, El-Ghanem M, et al. Spontaneous bilateral carotid-cavernous fistulas secondary to cavernous sinus thrombosis. Neurosurgery 2017; 80(4):646-54.

59. Say EAT, Shields CL, Bianciotti C, Shields JA. Chronic conjunctival chemosis from amlodipine besylate (norvasc). Cornea 2011;30:604-7.

60. Williams ZR. Carotid-cavernous sinus fistulae: a review of clinical presentation, therapeutic options, and visual prognosis. Int Ophthalmol Clin 58(2):271-94.

61. Desa V, Green R. Cavernous sinus thrombosis: current therapy. J Oral Maxillofac Surg 2012 Sep;70(9):2085-9162. Buckley EG. Cerebrovascular abnormalities. In: Wright KW, ed. Textbook of Ophthalmology. Baltimore, MD: Williams \& Wilkins, 1997:225-30.

63. Liu D. Conjunctivochalasis. Ophthalmic Plast Reconstruct Surg 1986;2:25-8.

64. Tse DT; Scott KR. The lacrimal system. In: Wright KW, ed. Textbook of Ophthalmology. Baltimore, MD: Williams \& Wilkins, 1997:367-89.
65. Welch J, Srinivasan S, Lyall D, Roberts F. Conjunctival lymphangiectasia: A report of 11 cases and review of literature. Surv Ophthalmol 2012;57(2):136-49.

66. Volek A, Toth J, Nagy ZZ, Schneider M. Evaluation of lymphatic vessel dilatations by anterior segment swept-source optical coherence tomography: case report. BMC Ophthalmol 2017;17:194

67. Anders H, Keller C. Pemberton's maneuver - a clinical test for latent superior vena cava syndrome caused by a substernal mass. Eur J Med Res 1997;2:488-90.

68. Chapel TA. The variability of syphilitic chancres. Sex Transm Dis 1978;5:68-70.

69. Mindel A, Tovey SJ, Timmins DJ, Willaims P. Primary and secondary syphilis, 20 years' experience. 2. Clinical features. Genitourin Med 1989 Jan;65(1):1-3.70. Chapel TA. The signs and symptoms of secondary syphilis. Sex Transm Dis 1980;7(4):161-4.

71. Campisi D, Whitcomb C. Liver disease in early syphilis. Arch Intern Med 1979; 139:365-6. 